\title{
CLASSICAL NOVAE IN ANDROMEDA: LIGHT CURVES FROM THE PALOMAR TRANSIENT FACTORY AND GALEX
}

\author{
Yi CaO $^{1,2}$, Mansi M. Kasliwal ${ }^{2,3}$, James D. Neill ${ }^{2}$, S. R. Kulkarni ${ }^{2}$, Yu-Qing Lou ${ }^{1}$, Sagi Ben-Ami ${ }^{4}$, Joshua S. Bloom ${ }^{5}$, \\ S. Bradley Cenko ${ }^{5}$, Nicholas M. Law ${ }^{6}$, Peter E. Nugent ${ }^{5,7}$, Eran O. Ofek ${ }^{8}$, Dovi Poznanski ${ }^{9}$, and Robert M. Quimby ${ }^{2,10}$ \\ ${ }^{1}$ Tsinghua Center for Astrophysics (THCA), Department of Physics, Tsinghua University, Beijing 100084, China; ycao@ astro.caltech.edu \\ ${ }^{2}$ Astronomy Department, California Institute of Technology, 1200 East California Boulevard, Pasadena, CA 91125, USA \\ ${ }^{3}$ Observatories of the Carnegie Institution for Science, 813 Santa Barbara Street, Pasadena, CA 91101, USA \\ ${ }^{4}$ Department of Particle Physics and Astrophysics, The Weizmann Institute of Science, Rehovot 76100, Israel \\ ${ }^{5}$ Department of Astronomy, University of California, Berkeley, CA 94720-3411, USA \\ ${ }^{6}$ Dunlap Institute for Astronomy and Astrophysics, University of Toronto, 50 St. George Street, Toronto, M5S 3H4 Ontario, Canada \\ ${ }^{7}$ Computational Cosmology Center, Lawrence Berkeley National Laboratory, 1 Cyclotron Road, Berkeley, CA 94720, USA \\ ${ }^{8}$ Benoziyo Center for Astrophysics, Faculty of Physics, The Weizmann Institute of Science, Rehovot 76100, Israel \\ ${ }^{9}$ School of Physics and Astronomy, Tel-Aviv University, Tel Aviv 69978, Israel \\ ${ }^{10}$ IPMU, University of Tokyo, Kashiwanoha 5-1-5, Kashiwa-shi, Chiba, Japan \\ Received 2012 January 12; accepted 2012 April 16; published 2012 June 5
}

\begin{abstract}
We present optical light curves of 29 novae in M31 during the 2009 and 2010 observing seasons of the Palomar Transient Factory (PTF). The dynamic and rapid cadences in PTF monitoring of M31, from one day to ten minutes, provide excellent temporal coverage of nova light curves, enabling us to record the photometric evolution of M31 novae in unprecedented detail. We also detect eight of these novae in the near-ultraviolet (UV) band with the Galaxy Evolution Explorer (GALEX) satellite. Novae M31N 2009-10b and M31N 2010-11a show prominent UV emission peaking a few days prior to their optical maxima, possibly implying aspherical outbursts. Additionally, our blueshifted spectrum of the recent outburst of PT And (M31N 2010-12a) indicates that it is a recurrent nova in M31 and not a dwarf nova in the Milky Way as was previously assumed. Finally, we systematically searched for novae in all confirmed globular clusters (GCs) of M31 and found only M31N 2010-10f associated with Bol 126. The specific nova rate in the M31 GC system is thus about one per year, which is not enhanced relative to the rate outside the GC system.
\end{abstract}

Key words: globular clusters: general - novae, cataclysmic variables - surveys

Online-only material: color figures

\section{INTRODUCTION}

Classical novae $(\mathrm{CNe})$ are interesting to study for at least two reasons: first, they provide a laboratory to investigate thermonuclear runaways in semidegenerate conditions (e.g., Bode \& Evans 2008); and second, they have been proposed as progenitors of Type Ia supernovae (e.g., Starrfield et al. 1988). Observations of Galactic novae are limited due to extinction and distance determination. Given the fact that nova outbursts are about $10^{3}-10^{4}$ times more frequent than supernovae and that the former are about $10^{3}-10^{4}$ times less luminous than the latter, nova searches are limited to the nearest galaxies.

CNe in M31 (The Andromeda Galaxy) have been observed for nearly a century (Hubble 1929; Arp 1956; Rosino 1964, 1973; Ciardullo et al. 1987; Rosino et al. 1989; Sharov \& Alksnis 1991; Tomaney \& Shafter 1992; Rector et al. 1999; Cassatella et al. 2004; Shafter \& Irby 2001; Darnley et al. 2004, 2006; Kasliwal et al. 2011; Shafter et al. 2011a). The published nova rates for M31 range between $24 \mathrm{yr}^{-1}$ (Arp 1956) and $65 \mathrm{yr}^{-1}$ (Darnley et al. 2004).

The Palomar Transient Factory (PTF; Law et al. 2009; Rau et al. 2009) acquired nearly daily monitoring of M31 in the $R$ band during the 2009 and 2010 observing seasons. It provides three advantages for the study of novae in M31: first, the large field of view (FoV) of $7.2 \mathrm{deg}^{2}$ covers the main region of M31 in one snapshot and with a spatial resolution of 1".0. Second, PTF has fast and dynamic cadences from one day to as short as ten minutes. This temporal coverage gives unprecedentedly detailed light curves. Third, the limiting magnitude is deeper than 20 mag (a little worse near the center of M31 where the background is strong and the gradient is steep). Hence, given the mean distance modulus of 24.36 for M31 (Vilardell et al. 2010), PTF can detect all but the most heavily extincted novae in M31 (weather-permitting) and follow them up down to a magnitude of $M_{R} \sim-4.4$.

These data also afford the opportunity to explore known recurrent novae ( $\mathrm{RNe}$ ) in $\mathrm{M} 31$ and perhaps discover new ones. According to the catalog maintained by Pietsch (2010), about 13 $\mathrm{RNe}$ have been discovered in M31. RNe are possible progenitors of Type Ia supernovae, since their white dwarf mass is thought to be closer to the Chandrasekhar mass limit than ordinary $\mathrm{CNe}$ (Starrfield et al. 1988).

Another issue related to novae that can be assessed with PTF archives is whether the nova rate in the globular cluster (GC) system is enhanced relative to that outside the GC system. Ciardullo et al. (1990b) did a search for novae in 54 M31 GCs based on the $\mathrm{H} \alpha$ surveys of Ciardullo et al. (1987, 1990a). In a mean effective survey time of approximately two years, they did not find any nova outbursts in any of the M31 GCs. Tomaney et al. (1992) reported another one-year search of over two hundred M31 GCs with $\mathrm{H} \alpha$ emission but did not find any nova outbursts, providing an upper limit on the rate of 0.005 nova $\mathrm{yr}^{-1} \mathrm{GC}^{-1}$. The only GC nova found in M31 is M31N 2007-06b (Shafter \& Quimby 2007). Henze et al. (2009a) then derived a rate of 1.1 nova $\mathrm{yr}^{-1}$ for the M31 GC system, which is comparable to the rate (per unit mass) outside the globulars. A systematic search for novae in M31 GCs using the PTF archives is presented here and also supports this conclusion. 
Table 1

Observation Summary

\begin{tabular}{lcc}
\hline \hline & PTF & GALEX \\
\hline Filter & Mould R & NUV \\
$\lambda_{\text {effect }}(\AA)$ & 6581 & 2316 \\
$\Delta \lambda(\AA)$ & 1251 & 1060 \\
$t_{\text {expose }}(\mathrm{s})$ & 60 & $740-1703$ \\
FoV $\left(\right.$ deg $\left.^{2}\right)$ & 7.2 & 1.2 \\
Cadence & 10 minutes to days & $1-2$ days \\
Epoch & 726 & 51 (the center field) \\
& & $22-27$ (outskirt fields) \\
\hline
\end{tabular}

In a nova outburst, except for a short "fireball" phase where radiation is given out by the thermal emission of the photosphere, UV photons are thought to originate at the surface of the white dwarf (WD), deep within the nova envelope, while optical photons are released near the surface of the envelope and result from absorption and re-emission of relatively low ionization species such as Cr II, Fe II, and other heavy elements in the envelope. These species are either from secular evolution of the WD progenitor or due to the mass transfer from the companion (Williams et al. 2008). Nova shells therefore serve as passive photon converters whose spectra reflect the reprocessing of incident UV light to longer wavelength bands. Eventually, the expansion decreases the temperature and dilutes absorbing species to finally reveal the UV photons (Kato \& Hachisu 1994; González-Riestra \& Krautter 1998). Therefore, to explore the UV light powering the nova emission, we searched in parallel for any contemporaneous UV observations of CNe in M31 with the Galaxy Evolution Explorer (GALEX) satellite (Martin et al. 2005). We obtained near UV-optical light curves for eight novae in M31.

This paper is arranged as follows. Section 2 describes PTF and $G A L E X$ observation and data reduction as well as data from other sources. Section 3 presents light curves of $\mathrm{CNe}$ and describes their morphology. Section 4 discusses novae in GCs. Section 5 focuses on RNe. Section 6 discusses ordinary and bizarre novae observed in the UV band. Section 7 revisits the relation between the maximum magnitude and the rate of decline (MMRD) with our nova sample. Finally, we conclude this paper in Section 8.

\section{OBSERVATION}

\subsection{Nova Sample}

We examined all novae as reported by Pietsch $(2010)^{11}$ in the PTF image archives and obtained the light curves of 29 novae. Among them, eight novae also have UV light curves from GALEX. Table 1 gives the main parameters of PTF and GALEX observations. Table 2 describes the nova sample and Figure 1 illustrates their spatial distribution.

\subsection{PTF}

PTF (Law et al. 2009) employs the $1.2 \mathrm{~m}$ Oschin Telescope at Palomar Observatory with an array of 12 CCD chips, each having $4 \mathrm{~K} \times 2 \mathrm{~K}$ pixels. One of the chips does not function for unknown reasons. The pixel size is $1^{\prime \prime} .0 \mathrm{pixel}^{-1}$ and thus the total FoV is $7.2 \mathrm{deg}^{2}$.

\footnotetext{
11 http://www.mpe.mpg.de/ m31 novae/opt/m31/index.php. This Web site
} maintains a comprehensive catalog of novae in M31.

\subsubsection{Survey Observation}

The time coverage of PTF observations of M31 during the 2009 and 2010 seasons is shown in Figure 2. The first observing season is from 2009 August 18 to 2010 January 17 and the second is from 2010 June 30 to 2010 December 15. From 2010 August 28 to 2010 September 7, PTF observed M31 with a fast cadence of about 10 minutes. All PTF images were taken with an $R$-band filter. The average seeing during the two seasons is 2 .'5 with a median value of 2 . 4 . We removed less than $1 \%$ of the images with seeing worse than 3 .'5, because the high stellar density in M31 leads to strong overlaps among adjacent objects under bad seeing conditions. Our final image database samples over seven hundred epochs on 7987 individual CCD images.

\subsubsection{Data Reduction and Photometry}

All images were processed through the PTF pipeline at the Lawrence Berkeley National Laboratory (see Law et al. 2009), obtaining astrometric solutions of 1".0 accuracy. The calibration of relative photometry proceeded as follows. We first use SExtractor to make a source catalog for each image. We then choose the catalog with the largest number of $14 \mathrm{mag}<R<$ 17 mag sources as reference. Then, other non-reference catalogs are spatially matched to the reference using source positions and forced to agree with the reference photometry after non-constant sources are removed with a standard outlier procedure. ${ }^{12}$ The resulting photometric uncertainty is usually around $0.05 \mathrm{mag}$. The absolute calibration is achieved by matching the reference sources to sources in the catalog presented in Massey et al. (2006).

Photometry is also done with SExtractor. The background is estimated in a region of $20^{\prime \prime} \times 20^{\prime \prime}$ centered at each target. Fluxes are measured within a Kron-like elliptical aperture. The firstorder moment of each object determines the Kron radius $r$ (Kron 1980) and the second-order moment determines ellipticity and position angle. The aperture has an area of $6.25 \pi r^{2}$. For a null detection, a $3 \sigma$ limiting magnitude is estimated.

In order to assess the quality of photometric results from SExtractor, we constructed light curves of three novae (M31N 200909a, M31N 2009-10a, and M31N 2009-11a) with three methods: (1) the one we described above, (2) aperture photometry with an aperture radius of seeing and with constant local background, and (3) aperture photometry with an aperture radius of seeing and with local background estimation by linear regression. All three methods show consistent magnitude measurements. Hence we adopt the first method.

\subsubsection{Photometric and Spectroscopic Follow-up}

Photometric follow-up of M31N 2010-11a was triggered for the Palomar 60 inch telescope (Cenko et al. 2006) on 2010 December 4. On 2010 December 30, we also used the Low Resolution Imaging Spectrometer (LRIS; Oke et al. 1995) mounted on the Keck I telescope to obtain spectra of M31N 2010-12a and M31N 2010-12c. These spectra were obtained with a configuration of the 560 dichroic, the $400 / 3400$ grating in the blue side and the $400 / 8500$ grating in the red side with a central wavelength of $7800 \AA$. Both spectra are shown in Figure 3.

\footnotetext{
12 NIST/SMATCH e-Handbook of Statistical Methods at http://www.itl.nist.gov/div898/handbook/eda/section3/ea35h.htm
} 

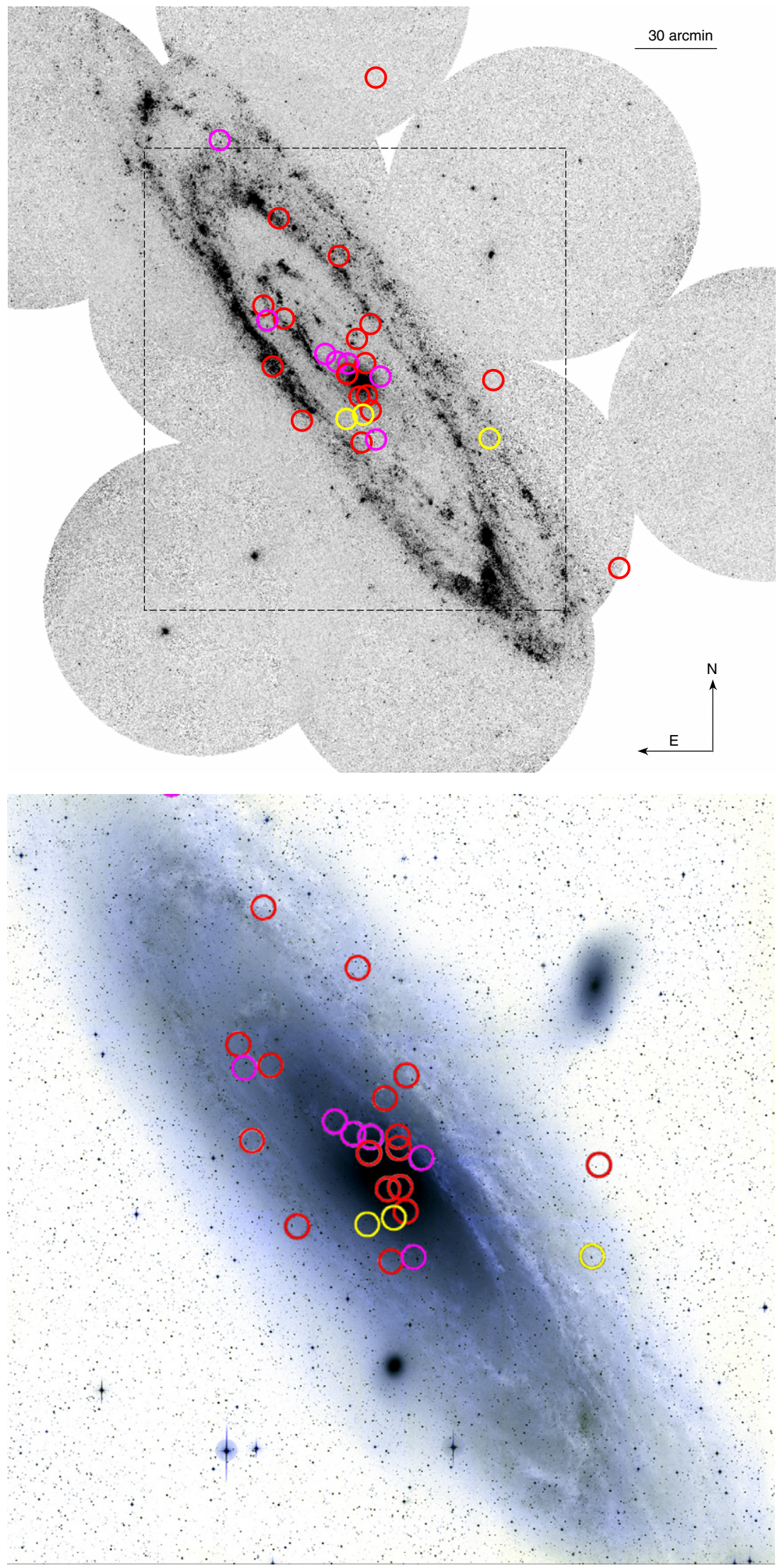

Figure 1. Spatial distribution of the nova sample in M31. The upper panel is an image of M31 taken by GALEX (Gil de Paz et al. 2007). The bottom panel is a mosaic image of M31 from PTF (Credit: P. Nugent). The dashed box in the upper panel shows the physical size of the bottom one. The actual FoV is larger than the size of this image. The red circles denote positions of novae only observed in PTF. RNe are highlighted by the yellow circles. The magenta circles denote novae observed by both PTF and GALEX.

(A color version of this figure is available in the online journal.) 
Table 2

Sample of Novae

\begin{tabular}{|c|c|c|c|c|c|c|c|}
\hline Nova Name & $\begin{array}{c}\alpha \\
(\mathrm{J} 2000)\end{array}$ & $\begin{array}{c}\delta \\
(\mathrm{J} 2000)\end{array}$ & $\begin{array}{l}\text { Discovery Date } \\
\text { by Others }\end{array}$ & $\begin{array}{l}\text { Magnitude } \\
\text { at Discovery }\end{array}$ & $\begin{array}{c}\text { PTF First Detection } \\
\text { Date }\end{array}$ & $\begin{array}{l}\text { Spectral } \\
\text { Type }\end{array}$ & Reference \\
\hline M31N 2009-08b & $00^{\mathrm{h}} 44^{\mathrm{m}} 09^{\mathrm{s}} .87$ & $+41^{\circ} 48^{\prime} 50^{\prime \prime} 8$ & $2009 / 08 / 09.780$ & $17.2(\mathrm{U})^{\mathrm{a}}$ & $2009 / 08 / 18.423$ & & 1 \\
\hline M31N 2009-08e & $00^{\mathrm{h}} 42^{\mathrm{m}} 36^{\mathrm{s}} .23$ & $+41^{\circ} 18^{\prime} 01^{\prime \prime} 6$ & $2009 / 08 / 25.896$ & 17.9(R) & $2009 / 08 / 26.400$ & Fe II & 2 \\
\hline M31N 2009-09a & $00^{\mathrm{h}} 42^{\mathrm{m}} 26^{\mathrm{s}} .08$ & $+41^{\circ} 04^{\prime} 01^{\prime \prime} 0$ & $2009 / 09 / 02.078$ & $17.1(\mathrm{U})$ & $2009 / 08 / 18.454$ & Fe II & 3 \\
\hline M31N 2009-10a & $00^{\mathrm{h}} 45^{\mathrm{m}} 14^{\mathrm{s}} .04$ & $+42^{\circ} 04^{\prime} 38^{\prime \prime} .8$ & $2009 / 10 / 03.619$ & $17.1(\mathrm{U})$ & $2009 / 09 / 30.494$ & Fe II & 4 \\
\hline M31N 2009-10b & $00^{\mathrm{h}} 42^{\mathrm{m}} 20^{\mathrm{s}} .77$ & $+41^{\circ} 16^{\prime} 44^{\prime \prime} .5$ & $2009 / 10 / 11.414$ & $18.8(\mathrm{R})$ & $2009 / 10 / 10.376$ & $\mathrm{Fe}$ II & 5 \\
\hline M31N 2009-11a & $00^{\mathrm{h}} 43^{\mathrm{m}} 04^{\mathrm{s}} .76$ & $+41^{\circ} 41^{\prime} 08^{\prime \prime} .2$ & $2009 / 11 / 03.559$ & $17.8(\mathrm{U})$ & $2009 / 10 / 31.321$ & $\mathrm{Fe}_{\mathrm{II}}$ & 6 \\
\hline M31N 2009-11bb & $00^{\mathrm{h}} 42^{\mathrm{m}} 39^{\mathrm{s}} 61$ & $+41^{\circ} 09^{\prime} 03^{\prime \prime} .2$ & $2009 / 11 / 06.523$ & $18.6(\mathrm{R})$ & $2009 / 11 / 07.199$ & $\ldots$ & 7 \\
\hline M31N 2009-11d & $00^{\mathrm{h}} 44^{\mathrm{m}} 16.85$ & $+41^{\circ} 18^{\prime} 53^{\prime \prime} .6$ & $2009 / 11 / 19.194$ & $18.1(\mathrm{R})$ & $2009 / 12 / 01.201$ & Fe II & 8 \\
\hline M31N 2009-11e & $00^{\mathrm{h}} 42^{\mathrm{m}} 35^{\mathrm{s}} \cdot 31$ & $+41^{\circ} 12^{\prime} 59^{\prime \prime} 1$ & $2009 / 11 / 21.138$ & 18.8(Swift uvw2) & $2009 / 12 / 01.201$ & $\mathrm{Fe}$ II & 9 \\
\hline M31N 2009-12a & $00^{\mathrm{h}} 40^{\mathrm{m}} 19^{\mathrm{s}} .40$ & $+41^{\circ} 15^{\prime} 47^{\prime \prime} \cdot 6$ & $2009 / 12 / 22.488$ & $16.6(\mathrm{U})$ & $2009 / 12 / 24.078$ & $\ldots$ & 10 \\
\hline$M 31 N 2010-01 a^{c}$ & $00^{\mathrm{h}} 42^{\mathrm{m}} 56^{\mathrm{s}} .74$ & $+41^{\circ} 17^{\prime} 21^{\prime \prime} .0$ & $2010 / 01 / 11.13$ & $17.6(\mathrm{R})$ & $2010 / 01 / 11.130$ & $\mathrm{Fe}$ II & 11 \\
\hline M31N 2010-06a & $00^{\mathrm{h}} 43^{\mathrm{m}} 07^{\mathrm{s}} .56$ & $+41^{\circ} 19^{\prime} 49^{\prime \prime} 0$ & $2010 / 06 / 28.014$ & $18.1(\mathrm{R})$ & $2010 / 06 / 30.397$ & Fe II & 12 \\
\hline M31N 2010-06b & $00^{\mathrm{h}} 44^{\mathrm{m}} 22^{\mathrm{s}} .46$ & $+41^{\circ} 28^{\prime} 14^{\prime \prime} .5$ & $2010 / 06 / 28.014$ & 19.1(R) & $2010 / 06 / 30.353$ & Fe II & 13 \\
\hline M31N 2010-06c & $00^{\mathrm{h}} 44^{\mathrm{m}} 04^{\mathrm{s}} .48$ & $+41^{\circ} 28^{\prime} 34^{\prime \prime} \cdot 2$ & $2010 / 06 / 26.084$ & $17.8(\mathrm{R})$ & $2010 / 06 / 30.353$ & $\ldots$ & 14 \\
\hline M31N 2010-06d & $00^{\mathrm{h}} 42^{\mathrm{m}} 55^{\mathrm{s}} \cdot 61$ & $+41^{\circ} 19^{\prime} 26^{\prime \prime} 0$ & $2010 / 06 / 24.02$ & 19.5(Swift uvw1) & $2010 / 07 / 04.397$ & Fe II & 15 \\
\hline M31N 2010-07a & $00^{\mathrm{h}} 43^{\mathrm{m}} 20^{\mathrm{s}} .11$ & $+41^{\circ} 21^{\prime} 23^{\prime \prime} \cdot 7$ & $2010 / 07 / 07.036$ & 20.6(Swift uvw1) & $2010 / 07 / 07.328$ & $\mathrm{Fe}_{\text {II }}$ & 16 \\
\hline M31N 2010-09a & $00^{\mathrm{h}} 42^{\mathrm{m}} 23^{\mathrm{s}} .32$ & $+42^{\circ} 17^{\prime} 08^{\prime \prime} .6$ & $2010 / 09 / 04.552$ & $17.2(\mathrm{U})$ & $2010 / 09 / 01.372$ & Fe II & 17 \\
\hline$M 31 N 2010-09 b$ & $00^{\mathrm{h}} 43^{\mathrm{m}} 45^{\mathrm{s}} .53$ & $+41^{\circ} 07^{\prime} 54^{\prime \prime} .7$ & 2010/09/30.412 & $17.7(\mathrm{R})$ & $2010 / 09 / 30.230$ & Fe II & 18 \\
\hline$M 31 N$ 2010-09c & $00^{\mathrm{h}} 38^{\mathrm{m}} 09^{\mathrm{s}} .06$ & $+40^{\circ} 37^{\prime} 25^{\prime \prime} .9$ & $2010 / 09 / 03.30$ & $18.7(\mathrm{U})$ & $2010 / 08 / 13.235$ & Fe II & 19 \\
\hline M31N 2010-10a & $00^{\mathrm{h}} 42^{\mathrm{m}} 45^{\mathrm{s}} .84$ & $+41^{\circ} 24^{\prime} 22^{\prime \prime} .2$ & $2010 / 10 / 05.551$ & $17.6(\mathrm{R})$ & $2010 / 10 / 08.163$ & Fe II & 20 \\
\hline M31N 2010-10b & $00^{\mathrm{h}} 42^{\mathrm{m}} 41^{\mathrm{s}} .51$ & $+41^{\circ} 03^{\prime} 27^{\prime \prime} \cdot 3$ & $2010 / 08 / 19.055$ & $18.9(\mathrm{R})$ & $2010 / 10 / 08.163$ & Fe II & 21 \\
\hline M31N 2010-10c & $00^{\mathrm{h}} 44^{\mathrm{m}} 26^{\mathrm{s}} .56$ & $+41^{\circ} 31^{\prime} 13^{\prime \prime} .8$ & $2010 / 10 / 13.557$ & $17.8(\mathrm{U})$ & $2010 / 10 / 13.113$ & Fe II & 22 \\
\hline M31N 2010-10d & $00^{\mathrm{h}} 42^{\mathrm{m}} 36^{\mathrm{s}} .91$ & $+41^{\circ} 19^{\prime} 29^{\prime \prime} 6$ & $2010 / 10 / 29.478$ & $17.8(\mathrm{U})$ & $2010 / 10 / 29.213$ & Fe II & 23 \\
\hline $\mathrm{M} 31 \mathrm{~N} 2010-10 \mathrm{e}^{\mathrm{d}}$ & $00^{\mathrm{h}} 42^{\mathrm{m}} 57^{\mathrm{s}} .76$ & $+41^{\circ} 08^{\prime} 12^{\prime \prime} .3$ & $2010 / 10 / 31.727$ & $18.1(\mathrm{R})$ & $2010 / 11 / 01.158$ & $\mathrm{He} / \mathrm{N}$ & 24 \\
\hline M31N 2010-10f & $00^{\mathrm{h}} 42^{\mathrm{m}} 43^{\mathrm{s}} .58$ & $+41^{\circ} 12^{\prime} 42^{\prime \prime} \cdot 6$ & 2010/11/07.06 & 0.02 counts $\mathrm{s}^{-1}(\mathrm{XRT})$ & $2010 / 10 / 12.189$ & $\ldots$ & 25 \\
\hline M31N 2010-11a & $00^{\mathrm{h}} 42^{\mathrm{m}} 31^{\mathrm{s}} .60$ & $+41^{\circ} 09^{\prime} 51^{\prime \prime} .5$ & $2010 / 11 / 23.05$ & 18.2(Swift uvw1) & $2010 / 12 / 01.250$ & $\mathrm{He} / \mathrm{N}$ & 26 \\
\hline$M 31 N 2010-12 a^{f}$ & $00^{\mathrm{h}} 40^{\mathrm{m}} 24^{\mathrm{s}} .37$ & $+41^{\circ} 04^{\prime} 03^{\prime \prime} .5$ & $2010 / 12 / 01.15$ & $16.7(\mathrm{U})$ & $2010 / 12 / 01.270$ & Fe II & 27 \\
\hline $\mathrm{M} 31 \mathrm{~N} 2010-12 \mathrm{~b}$ & $00^{\mathrm{h}} 42^{\mathrm{m}} 31^{\mathrm{s}} .08$ & $+41^{\circ} 27^{\prime} 20^{\prime \prime} 3$ & $2010 / 12 / 11.095$ & $16.7(\mathrm{U})$ & $2010 / 12 / 10.115$ & $\ldots$ & 28 \\
\hline $\mathrm{M} 31 \mathrm{~N} 2010-12 \mathrm{c}^{\mathrm{c}}$ & $00^{\mathrm{h}} 42^{\mathrm{m}} 56^{\mathrm{s}} \cdot 67$ & $+41^{\circ} 17^{\prime} 21^{\prime \prime} .2$ & $2010 / 12 / 15.528$ & $17.2(\mathrm{U})$ & $2010 / 12 / 15.093$ & $\mathrm{He} / \mathrm{N}$ & 29 \\
\hline
\end{tabular}

Notes.

${ }^{a}$ The bracket following the magnitude indicates the observation band and $\mathrm{U}$ represents unfiltered observation.

${ }^{\mathrm{b}} \mathrm{RN} ; 1997-11 \mathrm{k}=2001-12 \mathrm{~b}=2009-11 \mathrm{~b}$.

c According to CBET 2610, 2010-01a and 2010-12c are two different objects within 0.'8.

${ }^{\mathrm{d}} \mathrm{RN} ; 1963-09 \mathrm{c}=1968-09 \mathrm{a}=2001-07 \mathrm{~b}=2010-10 \mathrm{e}$.

e The nova 2010-10f is probably inside the GC Bol 126.

${ }^{\mathrm{f}}$ Another recurrent burst of PT And.

References. (1) Rodríguez-Gil et al. 2009; Ovcharov et al. 2009; (2) Ovcharov et al. 2009; Henze et al. 2009c; Medvedev et al. 2009; (3) Henze et al. 2009b; Barsukova et al. 2009b; (4) Fabrika et al. 2009b; (5) Nakano \& Yusa 2009; Podigachoski et al. 2009b; Hornoch \& Kusnirak 2009; Fabrika et al. 2009a; Hornoch et al. 2009a; Nakano et al. 2009; Nakano \& Itagaki 2009; Yamaoka et al. 2009; Martignoni et al. 2009; Di Mille et al. 2009; Barsukova et al. 2009a; Henze et al. 2009c; (6) Nishiyama \& Kabashima 2009a; Burwitz et al. 2009; Hornoch et al. 2009c; (7) Nishiyama et al. 2009a; Henze et al. 2009e; Kasliwal 2009; Henze et al. 2009d; Kasliwal et al. 2009; (8) Nishiyama \& Kabashima 2009b; Podigachoski et al. 2009d; Hornoch et al. 2009b; Podigachoski et al. 2009c; (9) Green 2009; Pietsch et al. 2009b; Cagas 2009; Kusnirak et al. 2009; Hornoch \& Pejcha 2009; Pietsch et al. 2009a; (10) Nishiyama et al. 2009b; (11) Burwitz et al. 2010c; Hornoch et al. 2010j, 2010i; Green 2010e; Pietsch \& Henze 2010a; Hornoch et al. 2010h; Burwitz et al. 2010d; Pietsch \& Henze 2010b; (12) Hornoch et al. 2010d, 2010e, 2010a, 2010k, 2010g; Henze et al. 2010c; Pietsch et al. 2010j; (13) Hornoch et al. 2010c, 2010a, 2010k; (14) Burwitz et al. 2010a; Pietsch et al. 2010d; Hornoch 2010; Hornoch et al. 2010a, 2010g; Burwitz et al. 2010b; (15) Pietsch et al. 2010e; Hornoch et al. 20101; Pietsch et al. 2010f; Henze et al. 2010c; Barsukova et al. 2010; Pietsch et al. 2010j; (16) Hornoch \& Zasche 2010; Henze et al. 2010a; Hornoch et al. 2010a, 2010k; Henze et al. 2010b, 2010c; Pietsch et al. 2010j; (17) Yusa 2010a; Shafter et al. 2010d; (18) Yusa 2010b; Pietsch et al. 2010k; Shafter et al. 2010f, 2010h; (19) Romadan et al. 2010; Shafter et al. 2010c; Krushinski et al. 2010; (20) Yusa 2010c; Shafter et al. 2010h; Pietsch et al. 2010j; (21) Corral-Santana et al. 2010; Shafter et al. 2010i; (22) Nakano \& Yusa 2010; Shafter et al. 2010g; (23) Nishiyama \& Kabashima 2010; Sun et al. 2010; Hornoch et al. 2010f; Hornochova \& Wolf 2010; Shafter et al. 2010b; (24) Hornochova et al. 2010; Pietsch et al. 2010b; Shafter et al. 2010a; Pietsch et al. 2010g (25) Pietsch et al. 2010i; Henze et al. 2010d; Shafter et al. 2010e; (26) Pietsch et al. 2010h; Cao et al. 2010; Fabrika et al. 2010; (27) Zheng et al. 2010; (28) Green 2010a; Koishikawa 2010; Pietsch et al. 2010a; Green 2010b, 2010c; Yusa 2010d; Koishikawa et al. 2010; Pietsch et al. 2010c; (29) Prieto et al. 2010a; Yusa 2010e; Green 2010d, 2010f; Prieto et al. 2010b.

\subsection{GALEX}

GALEX is a wide-field imaging UV space telescope that was originally launched with near-UV (NUV) and far-UV (FUV) detectors. Unfortunately, the FUV detector failed before the start of the PTF survey of M31. The NUV detector effective wavelength is $2316 \AA$ and the band width is $1060 \AA$ (Martin et al. 2005; Morrissey et al. 2007). This channel has an FoV of $1.2 \mathrm{deg}^{2}$ (Morrissey et al. 2007).

\subsubsection{GALEX Observation Summary}

The visibility of M31 for GALEX is defined by the Sun, Moon, and Earth-limb constraints that limit the observations more 


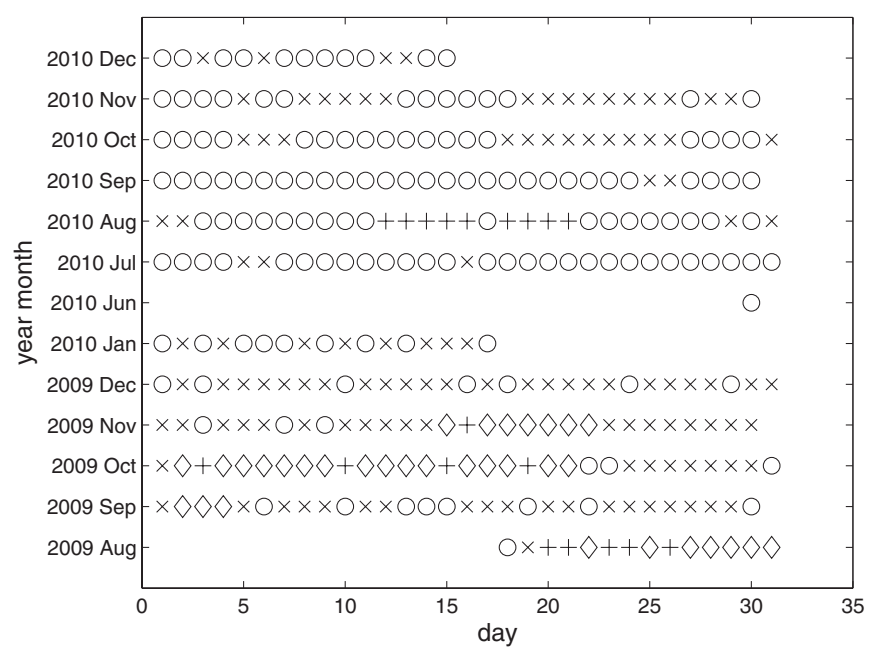

Figure 2. Temporal coverage of both PTF and GALEX. A plus sign indicates an epoch observed by both PTF and GALEX, while a cross indicates an epoch observed by neither. PTF-only observations are denoted with circles. GALEXonly observations are represented by diamonds. Statistically, during the first observing season, PTF observed M31 for $20 \%$ of 156 total nights, GALEX observed for $21 \%$ nights and the two overlapped on $6 \%$ nights. In the second season, PTF observed for $75 \%$ of 169 nights and GALEX overlapped with PTF on nine nights.

Table 3

Novae Observed with GALEX

\begin{tabular}{lccc}
\hline \hline Name & Epochs & UT Start & UT End \\
\hline M31N 2009-09a & 63 & $2009 / 08 / 20.429$ & $2009 / 10 / 15.707$ \\
M31N 2009-10a & 13 & $2009 / 10 / 02.629$ & $2009 / 10 / 21.457$ \\
M31N 2009-10b & 22 & $2009 / 10 / 08.587$ & $2009 / 11 / 15.725$ \\
M31N 2009-11b & 63 & $2009 / 08 / 20.429$ & $2009 / 11 / 15.725$ \\
M31N 2010-06a & 13 & $2010 / 08 / 12.894$ & $2010 / 08 / 21.998$ \\
M31N 2010-06b & 13 & $2010 / 08 / 12.894$ & $2010 / 08 / 21.998$ \\
M31N 2010-06d & 13 & $2010 / 08 / 12.894$ & $2010 / 08 / 21.998$ \\
M31N 2010-07a & 13 & $2010 / 08 / 12.894$ & $2010 / 08 / 21.998$ \\
\hline
\end{tabular}

than for ground-based observatories. The result is that GALEX was able to intensively monitor M31 in several campaigns that overlap with the PTF campaigns of 2009 and 2010 (2009 August 8 to 2009 September 4, 2009 October 2 to 2009 October 21, 2009 November 15 to 2009 November 22, and 2010 August 12 to 2010 August 21). Figure 2 illustrates GALEX temporal coverage and its overlap with PTF. A field centered in M31 is monitored daily, while ten outskirt fields in M31 are observed every other day. So we have 51 epochs of the center field while about 22-27 epochs of the outskirt fields. The exposures were all taken during single-orbit pointings and thus have durations close to $1500 \mathrm{~s}$ with a range from $740 \mathrm{~s}$ to $1703 \mathrm{~s}$. Table 1 summarizes parameters of observations.

\subsubsection{Photometry}

Photometry was done with an aperture with a radius of 6 arcsec at each nova position and was calibrated using the standard GALEX zero points to the $\mathrm{AB}$ magnitude system (Morrissey et al. 2007). Eight novae were detected; see Table 3.

\subsection{Data from Other Sources}

For completeness in optical and UV bands, we incorporate photometric measurements of novae from ATel and CBET reports (see the reference column in Table 2). This includes optical photometry in the $R, B, V, r^{\prime}, i^{\prime}$, and $\mathrm{H} \alpha$ bands as well
Table 4

X-Ray and Spectroscopic Observation Epochs of M31 Novae

\begin{tabular}{|c|c|c|c|}
\hline \multirow[t]{2}{*}{ Novae } & \multicolumn{2}{|c|}{ Observation UT } & \multirow[t]{2}{*}{ Reference } \\
\hline & Spectrum & X-Ray & \\
\hline M31N 2009-08e & $2009 / 09 / 18.014$ & & Medvedev et al. 2009 \\
\hline M31N 2009-09a & 2009/09/18.18 & & Barsukova et al. 2009b \\
\hline M31N 2009-10a & 2009/10/09.91 & & Fabrika et al. 2009b \\
\hline \multirow[t]{3}{*}{ M31N 2009-10b } & $2009 / 10 / 09.820$ & & Fabrika et al. 2009a \\
\hline & $2009 / 10 / 15.5$ & & Yamaoka et al. 2009 \\
\hline & 2009/10/19.936 & & Barsukova et al. 2009a \\
\hline M31N 2009-11a & $2009 / 11 / 25.32$ & & Hornoch et al. 2009c \\
\hline \multirow[t]{2}{*}{ M31N 2009-11b } & $2009 / 11 / 08.35$ & & Kasliwal 2009 \\
\hline & $2009 / 11 / 09.82$ & & Kasliwal et al. 2009 \\
\hline M31N 2009-11d & $2009 / 11 / 21.31$ & & Hornoch et al. $2009 b$ \\
\hline M31N 2009-11e & $2009 / 11 / 22.19$ & & Hornoch \& Pejcha 2009 \\
\hline \multirow[t]{2}{*}{ M31N 2010-01a } & $2010 / 01 / 13.08$ & & Hornoch et al. $2010 \mathrm{j}$ \\
\hline & $2010 / 01 / 15.09$ & & Hornoch et al. $2010 \mathrm{i}$ \\
\hline M31N 2010-06a & $2010 / 07 / 19$ & & Hornoch et al. $2010 \mathrm{~g}$ \\
\hline M31N 2010-06b & 2010/07/19 & & Hornoch et al. $2010 \mathrm{~g}$ \\
\hline M31N 2010-06d & $2010 / 08 / 08.86$ & & Barsukova et al. 2010 \\
\hline M31N 2010-07a & $2010 / 07 / 19$ & & Hornoch et al. $2010 \mathrm{~g}$ \\
\hline M31N 2010-09a & $2010 / 09 / 07.23$ & & Shafter et al. 2010d \\
\hline \multirow[t]{2}{*}{ M31N 2010-09b } & $2010 / 10 / 01.39$ & & Shafter et al. 2010f \\
\hline & $2010 / 10 / 06.40$ & & Shafter et al. $2010 \mathrm{~h}$ \\
\hline M31N 2010-09c & $2010 / 09 / 14.23$ & & Shafter et al. 2010c \\
\hline M31N 2010-10a & $2010 / 10 / 06.38$ & & Shafter et al. $2010 \mathrm{~h}$ \\
\hline \multirow[t]{3}{*}{ M31N 2010-10b } & $2010 / 10 / 28.31$ & & Shafter et al. 2010i \\
\hline & 2010/11/03.31 & & Shafter et al. 2010i \\
\hline & $2010 / 11 / 11.28$ & & Shafter et al. 2010i \\
\hline M31N 2010-10c & $2010 / 10 / 17.35$ & & Shafter et al. $2010 \mathrm{~g}$ \\
\hline M31N 2010-10d & $2010 / 10 / 30.31$ & & Shafter et al. 2010b \\
\hline \multirow[t]{3}{*}{ M31N 2010-10e } & $2010 / 11 / 04.32$ & & Shafter et al. 2010a \\
\hline & & $2010 / 11 / 15.16$ & Pietsch et al. $2010 \mathrm{~g}$ \\
\hline & & 2010/11/17.09 & Pietsch et al. $2010 \mathrm{~g}$ \\
\hline \multirow[t]{4}{*}{ M31N 2010-10f } & $2010 / 11 / 13.285$ & & Shafter et al. 2010e \\
\hline & & 2010/11/03.04 & Pietsch et al. 2010i \\
\hline & & $2010 / 11 / 06.20$ & Pietsch et al. 2010i \\
\hline & & 2010/11/07.06 & Pietsch et al. 2010i \\
\hline M31N 2010-11a & $2010 / 12 / 05.74$ & & Fabrika et al. 2010 \\
\hline M31N 2010-12a & $2010 / 12 / 30.24$ & & This paper \\
\hline M31N 2010-12c & $2010 / 12 / 30.25$ & & This paper \\
\hline
\end{tabular}

as unfiltered data. We also include data published in Shafter et al. (2011a) where several novae in 2009 are well sampled. The UV data in ATel reports are mainly obtained by Swift (Gehrels et al. 2004). For consistency of data between GALEX and Swift, all Swift data are calibrated onto the AB magnitude system (Siegel et al. 2010). When data from different sources are used, due to difference of filters, data may have systematical errors among different projects.

In addition, we include X-ray and spectroscopic information of novae in M31 from Swift from either ATel reports or our own analysis (see Table 4).

\subsection{Missed Novae}

During the two seasons presented here, we did not find three announced novae in the PTF archive: M31N 2009-10c, M31N 2009-11c, and M31N 2010-07b.

M31N 2009-10c took place at $\alpha=00^{\mathrm{h}} 42^{\mathrm{m}} 45^{\mathrm{s}} .8, \delta=$ $+41^{\circ} 15^{\prime} 57^{\prime \prime}$ (J2000), less than 30 arcsec from the center of M31. This nova reached maximum light, $R=17.2$, on 2009 October 9 (Podigachoski et al. 2009a). PTF observed M31 on 2009 October 10 . In the vicinity of the nova position, if we fit a constant background, pixels have on average $\sim 50,000$ with a standard deviation of $\sigma \sim 6000$. Given that the seeing then was 

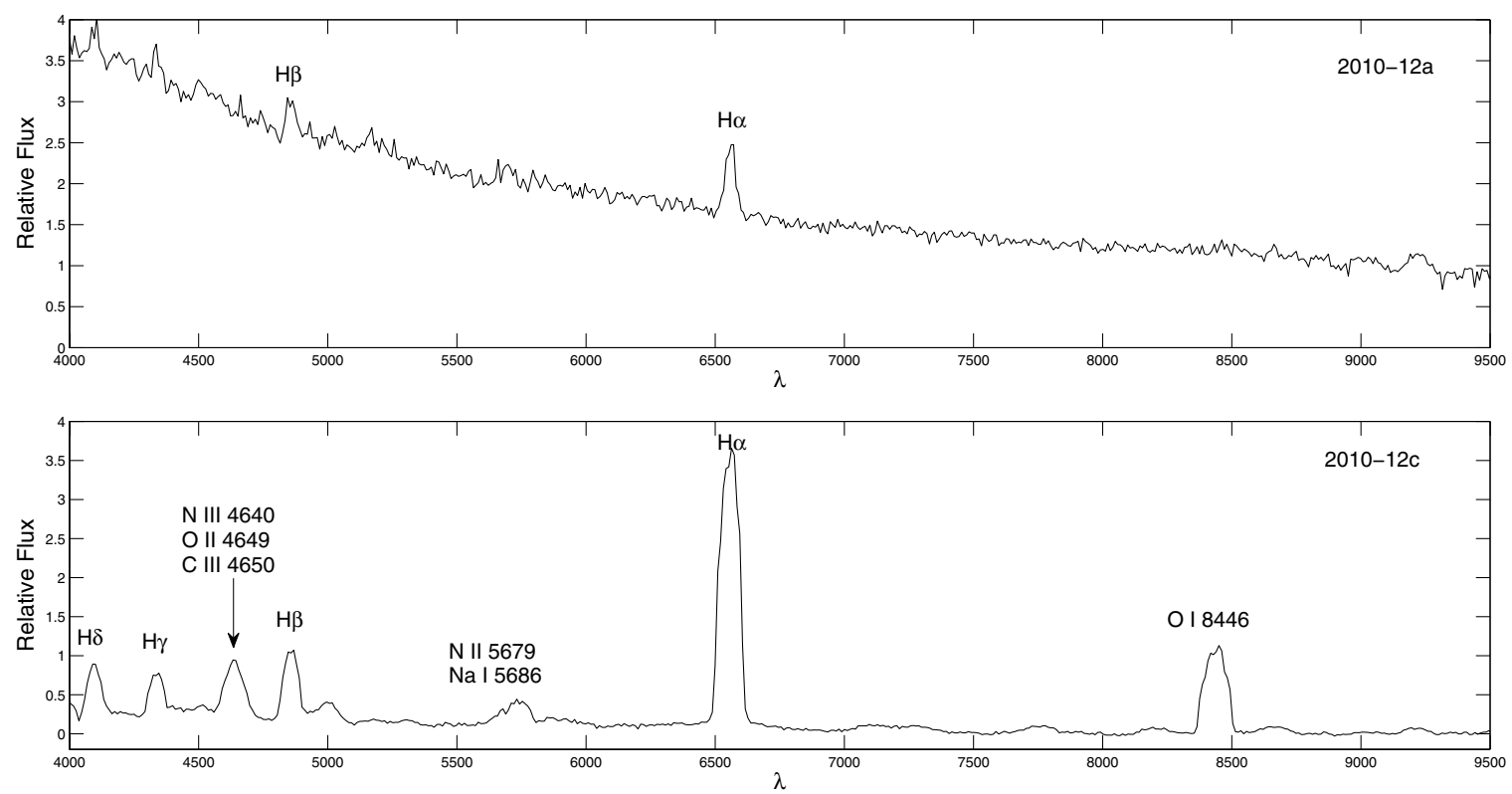

Figure 3. Spectra of M31N 2010-12a (top) and M31N 2010-12c (bottom), obtained with LRIS on the Keck I telescope on 2010 December 30.

about 3 arcsec and that the zero point is 27.6 , the $3 \sigma$ limiting magnitude is 15.2 . If the background is fit with a plane, the standard deviation is roughly 1500 and the limiting magnitude is 16.8. Thus, we did not detect this nova in the images. In the subtraction image, the circle of 30 arcsec in radius in the center of M31 is excluded in the subtraction algorithm where the algorithm does not work well. As a result, we missed this nova in our PTF data.

There are gaps of 40 arcsec between adjacent CCDs. M31N 2009-11c unfortunately fell in the gap between two adjacent CCDs.

M31N 2010-07b showed strong $\mathrm{H} \alpha$ emission in July. However, its luminosities in the $R$ and $r^{\prime}$ bands were about 20.7 mag in June and July (Hornoch et al. 2010b). This fact suggested that this nova likely peaked much earlier and decayed in June and July. The second observing season of PTF started at the end of June (see Figure 1). This nova thus was below our single-image detection threshold.

Admittedly, we did not carry out a real-time search of novae in M31 in the first two seasons of PTF. Our nova sample inherits the sensitivity and incompleteness of those projects that discovered these novae.

\subsection{Special Novae \\ 2.6.1. M31 2010-09c}

This nova has a faint and close neighbor with $R=19.85 \pm 0.2$ within 2 arcsec. In SDSS Data Release 8, we found two nearby sources within 2 arcsec. With the empirical relation between photometry of ugriz and UBVRI systems (Jordi et al. 2006), we obtain that the neighbors have $R=20.53$ and $R=20.33$, giving a total magnitude of 19.67 that is consistent with our measurements. The uncertainty caused by the neighbors has been included in the nova light curve. The spectroscopic confirmation in (Shafter et al. 2010c) identified M31N 2010$09 \mathrm{c}$ as a real nova of Fe II type.

\subsubsection{M31 2010-10f}

This nova is spatially associated with the M31 GC Bol 126 (Wirth et al. 1985). In Figure 4, we clearly see a brightening of the GC by about 1 mag on around 2010 October 10 (Julian Date: 2455480). The luminosity of the GC before the nova is $16.8 \pm 0.2$. The light curve of the nova is obtained by subtracting the GC brightness. One possible reason for the large uncertainty in the photometry measurements is that the nova is only a few pixels away from the edge of the CCD.

\subsubsection{M31N 2010-01a and M31N 2010-12c}

These two novae occurred within 1 arcsec of each other, a separation which is less than the pixel scale of PTF $\left(\simeq 1^{\prime \prime}\right.$. 0$)$. A highly accurate astrometric solution shows that they are indeed two separate objects (Green 2010f).

We obtained a spectrum of 2010-12c with LRIS on 2010 December 30 (Figure 3, bottom panel). The spectrum shows a weak continuum superposed with many emission lines, illustrating that the nova has already entered the nebular phase. We identified $\mathrm{H} \alpha, \mathrm{H} \beta, \mathrm{H} \gamma, \mathrm{H} \delta, \mathrm{O}$ I 8446, and several $\mathrm{He}$ and $\mathrm{N}$ lines. $\mathrm{H} \delta$ has a slight $\mathrm{P}$ Cygni profile. The emission lines suggest that the nova is of the He/ $\mathrm{N}$ type. After fitting each emission line with a Gaussian profile, we find that $\mathrm{H} \alpha$ is centered at $6556 \AA$, $\mathrm{H} \beta$ at $4858 \AA, \mathrm{H} \delta$ at $4338 \AA$, and O I 8446 at $8437 \AA$, suggesting a blueshifted velocity in the range from -200 to $-300 \mathrm{~km} \mathrm{~s}^{-1}$. From the full width at zero intensity (FWZI) of the Balmer lines, we derived an expansion velocity of $\simeq 3000 \mathrm{~km} \mathrm{~s}^{-1}$. On the other hand, the systemic velocity of M31 is $-300 \mathrm{~km} \mathrm{~s}^{-1}$. The nova lies about $2^{\prime}$ northeast of the center of M31. The H I $21 \mathrm{~cm}$ line (Chemin et al. 2009; Corbelli et al. 2010) suggests a rotation velocity of about $260 \mathrm{~km} \mathrm{~s}^{-1}$ receding from us. However, the nova is apparently close to the center of the galaxy and therefore may not share the motion of the disk measured by the $\mathrm{H}$ I $21 \mathrm{~cm}$ line. Another measurement of a nearby planetary nebula (Merrett et al. 2006) at $\alpha=00^{\mathrm{h}} 42^{\mathrm{m}} 57^{\mathrm{s}} .4$ and $\delta=+41^{\circ} 17^{\prime} 26^{\prime \prime}$ (J2000) suggests a radial velocity of $-300 \mathrm{~km} \mathrm{~s}^{-1}$ along the line of sight. Consequently, we conclude that $2010-12 \mathrm{c}$ is a real nova in M31.

Additionally, these two novae have two more historical neighboring novae within $10^{\prime \prime}$ : nova 30 (discovered in 1986) from Ciardullo et al. (1987) and M31N 2009-08a. Generally speaking, our sample includes more novae in the central region 

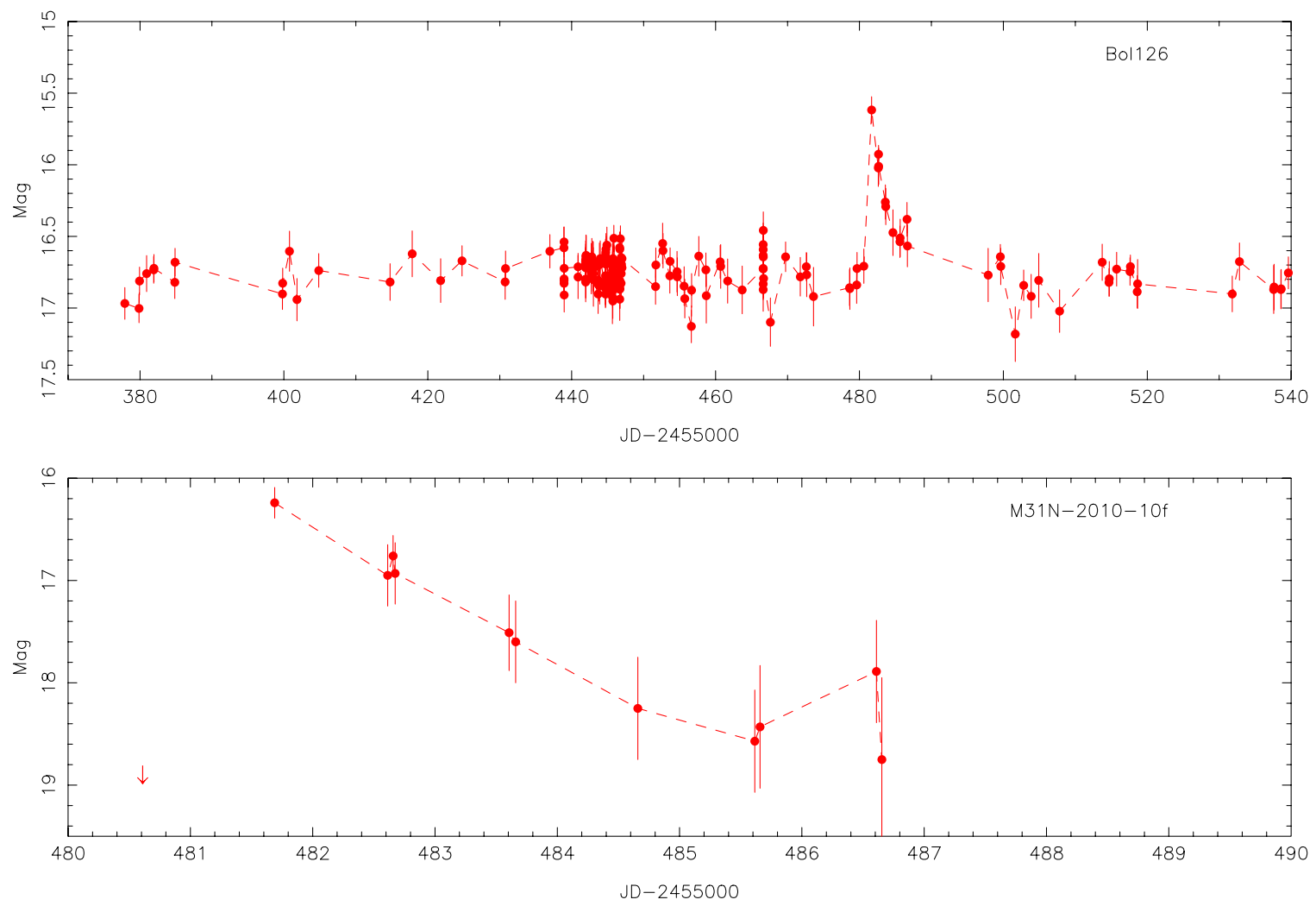

Figure 4. Light curve of the GC Bol 126 is shown in the top panel. The subtracted light curve of M31N 2010-10f is presented in the bottom panel.

(A color version of this figure is available in the online journal.)

of M31 than in the outskirt. This might result from the high stellar density in the center of the galaxy.

\section{LIGHT CURVES OF CLASSICAL NOVAE}

Except three recurrent events, light curves of $26 \mathrm{CNe}$ are presented in Figures 5-9. An electronic version of the PTF and GALEX data is online ${ }^{13}$ in a machine-readable format. An example of M31N 2010-12a is illustrated in Table 5.

The nova light curves are presented in groups based on their features described below. Figures 5 presents novae with smooth decline. Figure 6 illustrates novae with jittery decline. Novae with well-sampled rise stages are displayed in Figure 7. Figure 8 highlights two novae recorded during the PTF 10 minute cadence observations where their rise histories were recorded in great detail. Figure 4 shows the light curves of the only GC nova M31N 2010-10f and its host Bol 126. All other undersampled light curves are collected in Figure 9.

Table 6 summarizes the photometric and morphological features of these novae.

\subsection{Morphological Classification}

The shape of each light curve can be characterized by several parameters: the rise rate, the rise time to the maximum magnitude, the rise behavior, the maximum magnitude, the decline rate that is represented by $t_{n}(n=1,2,3, \ldots)$ defined as the time interval during which a nova decays from its maximum by $n$ magnitudes, and the decline behavior. Since PTF samples the M31 nova luminosity distribution down to $M_{R} \sim-4.5$ (see Section 1) and the peak absolute magnitude of a nova can

\footnotetext{
13 http://www.astro.caltech.edu/ ycao/m31 novae/index.html
}

Table 5

Photometric Measurements of M31N 2010-12a

\begin{tabular}{lcc}
\hline \hline Julian Date & Magnitude & Magnitude Error $^{\mathrm{a}}$ \\
\hline 2455530.702 & 20.190 & 99.000 \\
2455531.770 & 16.763 & 0.030 \\
2455532.780 & 15.581 & 0.023 \\
2455532.825 & 15.561 & 0.025 \\
2455535.681 & 16.013 & 0.024 \\
2455537.578 & 16.325 & 0.024 \\
2455537.622 & 16.346 & 0.025 \\
2455538.577 & 16.526 & 0.024 \\
2455538.621 & 16.496 & 0.025 \\
2455539.577 & 16.636 & 0.026 \\
2455539.620 & 16.638 & 0.025 \\
2455540.615 & 16.721 & 0.027 \\
2455540.659 & 16.776 & 0.026 \\
2455541.613 & 16.817 & 0.027 \\
2455541.656 & 16.899 & 0.031 \\
2455544.593 & 17.243 & 0.029 \\
2455544.639 & 17.175 & 0.032 \\
2455545.593 & 17.283 & 0.038 \\
2455545.637 & 17.303 & 0.034 \\
\hline
\end{tabular}

Note. ${ }^{a} 99.000$ in magnitude error indicates that a $3 \sigma$ upper limit is given in magnitude.

range from -5 to -9 , we use $t_{2}$ to indicate the decline rate. In our measurements, $t_{2}$ is estimated by linear interpolation between adjacent measurements. Other parameters are defined as follows: the rise rate is the mean rate from the first detection to the first local maximum; the rise time is the interval from first detection in PTF to its global maximum. All measurements are done with PTF $R$-band data. 

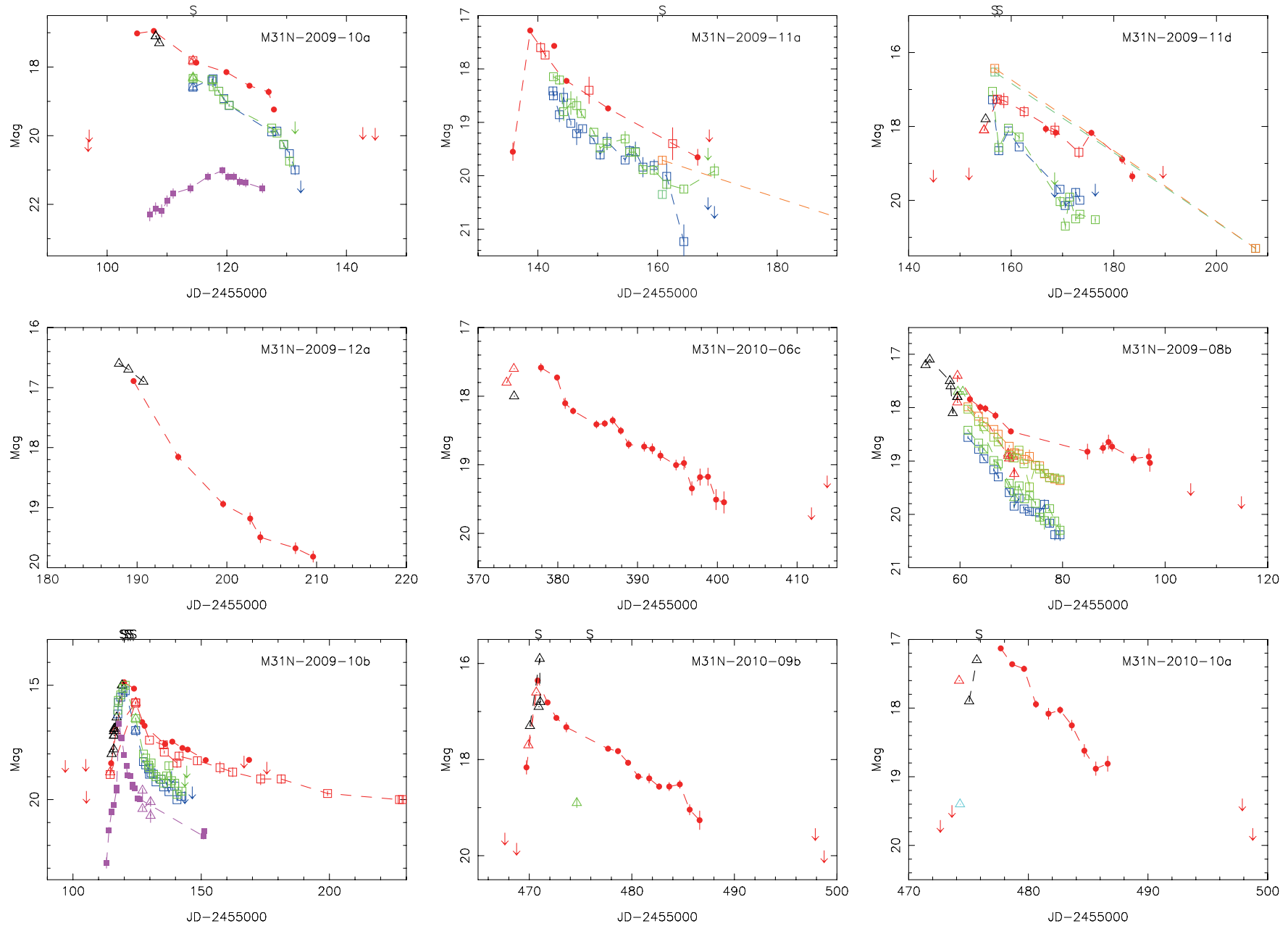

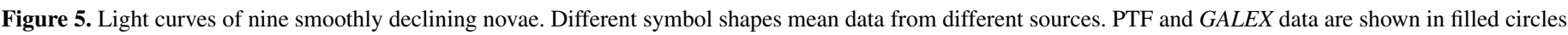

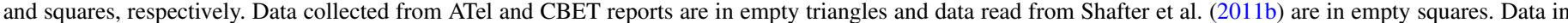

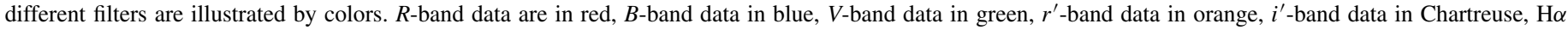

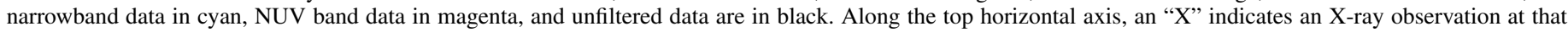
time and an " $\mathrm{S}$ " indicates a spectrum of the nova taken at that time.

(A color version of this figure is available in the online journal.)

For novae inside our Galaxy, Strope et al. (2010) classified the diverse light curves morphologically according to their evolution of decline. With a median coverage extending to 8.0 mag below peak, novae were grouped into seven classes. In their sample of 93 nova light curves, 38\% decline smoothly, $21 \%$ have plateau phase, $18 \%$ show dust dips, $1 \%$ rebrighten with a cusp-shaped secondary maxima, $4 \%$ oscillate quasi-sinusoidally on smooth decline, $2 \%$ are flat-topped, and $16 \%$ have irregular jitters or flared during decline. However, PTF is unable to follow M31 novae to such late times in the light curve evolution. We, instead, roughly classify our M31 novae into two major classes: S-class for smooth light curves and J-class for jittery light curves or for light curves with flares superposed on the decline. Statistically in our nova sample, $60 \%$ show S-class decline and $40 \%$ belong to the J-class. Given that our sample is quite small, this result is roughly consistent with the Galactic population. We do not see any flat-topped nova.

Similarly, due to the rapid cadences, PTF sampled the rising stages of several novae very well. This enables us to employ the same S-J classification to describe the rising parts of the light curves (see Table 6). We find that the S-class novae rise very fast (e.g., M31N 2010-09b rose within 2 days), while the J-class novae rise more slowly (e.g., M31N 2010-10b took 30 days to reach its maximum). But we do not see any relation between the rise time and peak magnitudes. Luminous M31N 2010-09b and subluminous M31N 2009-11a rose rapidly, while luminous M31N 2010-07a and subluminous M31N 2009-09a both rose very slowly.

During a very high cadence (tens of minutes) experiment in PTF, we observed the rising stages of M31N 2010-09a and M31N 2010-09c in unprecedented detail, as shown in the bottom panels of Figure 8 . The rise of both novae is smooth over a timescale of tens of minutes.

\subsection{Super-Eddington Phase}

Some novae around maxima exhibit super-Eddington phases. Theorists (e.g., Shaviv 2001) model this phase with a porous structure in the nova wind that reduces the effective opacity and raises the Eddington luminosity. Kato \& Hachisu (2005) produced a light curve model based on this idea that successfully matched the super-Eddington phase of Nova V1974 Cyg and demonstrated the importance of the relationship between the optical and UV light curves. They isolated a portion of the UV continuum near $1455 \AA$ that had a duration that was well correlated with the decline rate of the nova in the optical. 

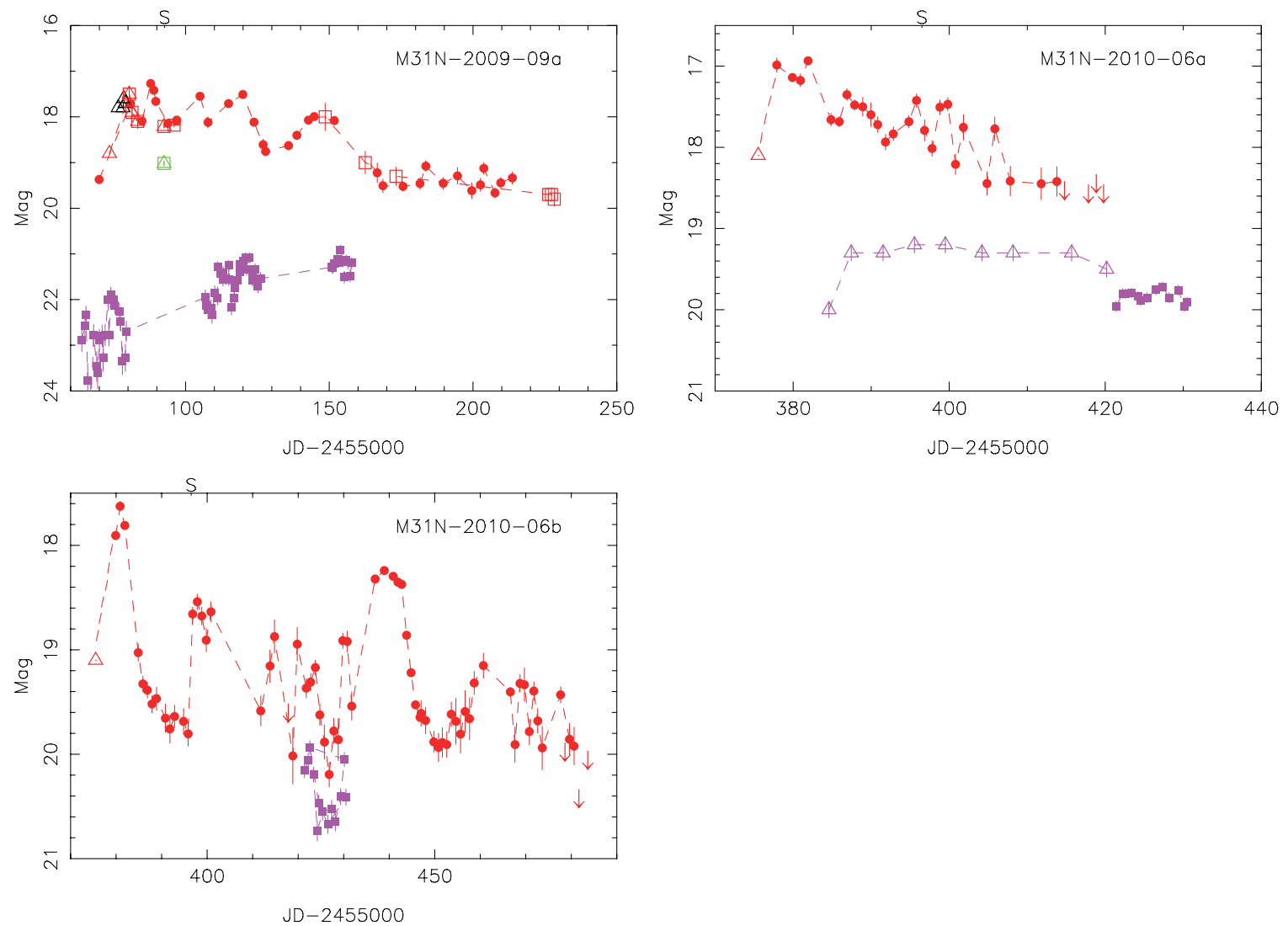

Figure 6. Light curves of novae with jittering decay. Symbol designations are the same as those in Figure 5.

(A color version of this figure is available in the online journal.)
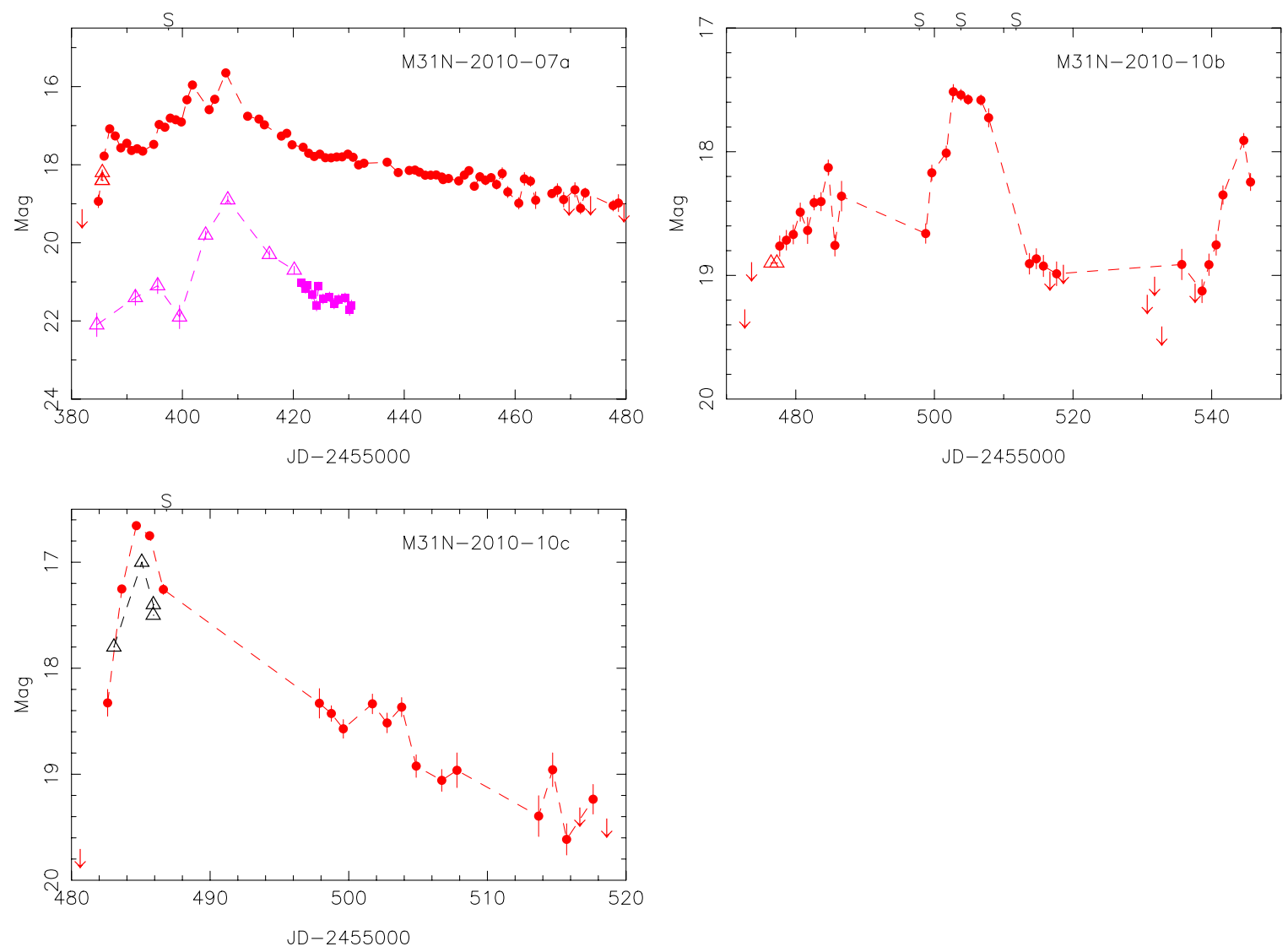

Figure 7. Light curves whose rise stages are well sampled. Symbol designations are the same as those in Figure 5.

(A color version of this figure is available in the online journal.) 

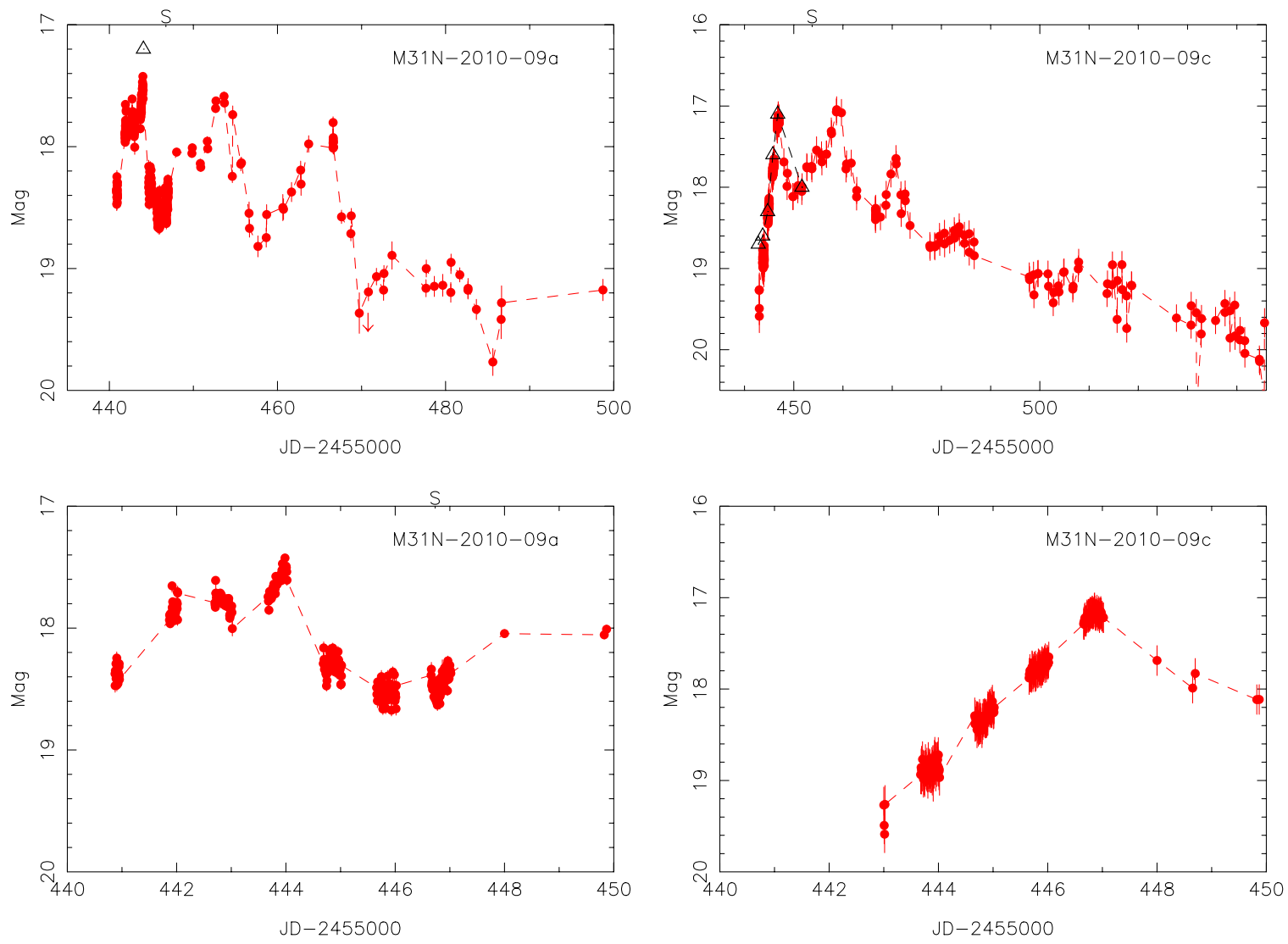

Figure 8. M31N 2010-09a and M31N 2010-09c were observed during the PTF 10 minute cadence experiments. In the top two panels, we show both full light curves. In the bottom two panels, rising phases are highlighted. Symbol designations are the same as those in Figure 5.

(A color version of this figure is available in the online journal.)

Table 6

Properties of PTF Well-sampled Nova Light Curves

\begin{tabular}{|c|c|c|c|c|c|c|}
\hline Nova & $\begin{array}{l}\text { Rising Rate } \\
\left(\mathrm{mag} \mathrm{day}^{-1}\right)\end{array}$ & $\begin{array}{l}\text { Rising Time } \\
\text { (days) }\end{array}$ & $\begin{array}{c}\text { Rising } \\
\text { Morphology }\end{array}$ & $\begin{array}{c}t_{2} \\
\text { (days) }\end{array}$ & $\begin{array}{c}\text { Decline } \\
\text { Morphology }\end{array}$ & $\begin{array}{c}R \text {-Band Peak } \\
\text { Magnitude }\end{array}$ \\
\hline 2009-08b & $\ldots$ & $\ldots$ & $\ldots$ & $>35$ & $\mathrm{~J}$ & 17.76 \\
\hline $2009-09 a$ & $\ldots$ & 26 & $\mathrm{~J}$ & 79 & $\mathrm{~J}$ & 17.26 \\
\hline 2009-10a & $\ldots$ & $\ldots$ & $\ldots$ & 20 & S & 16.95 \\
\hline $2009-10 b$ & 0.6 & 5 & $\mathrm{~S}$ & 8 & S & 14.84 \\
\hline 2009-11a & 0.8 & 3 & $\mathrm{~S}$ & 22 & $\mathrm{~S}$ & 17.23 \\
\hline $2009-11 b$ & $\ldots$ & $\ldots$ & $\ldots$ & $>37$ & $\mathrm{~J}$ & 18.25 \\
\hline $2009-11 d$ & $\ldots$ & $\ldots$ & $\ldots$ & $>17$ & $\mathrm{~J}$ & $17.72^{\mathrm{a}}$ \\
\hline $2009-12 a$ & $\ldots$ & $\ldots$ & $\ldots$ & 10 & $S$ & 16.88 \\
\hline $2010-06 a$ & $\ldots$ & $\ldots$ & $\ldots$ & $>31$ & $\mathrm{~J}$ & 16.88 \\
\hline 2010-06b & 0.4 & $\ldots$ & $\ldots$ & 8 & $\mathrm{~J}$ & 17.62 \\
\hline $2010-06 c$ & $\ldots$ & $\ldots$ & $\ldots$ & 19 & $S$ & 17.57 \\
\hline $2010-07 a$ & 0.7 & 26 & $\mathrm{~J}$ & 15 & S & 15.65 \\
\hline $2010-09 a$ & 0.5 & 14 & $\mathrm{~J}$ & 40 & $\mathrm{~J}$ & 17.42 \\
\hline $2010-09 b$ & 1.6 & 2 & S & 10 & $S$ & 16.36 \\
\hline $2010-09 c$ & 0.4 & 18 & $\mathrm{~J}$ & 57 & $\mathrm{~J}$ & 17.04 \\
\hline $2010-10 \mathrm{a}$ & $\ldots$ & $\ldots$ & $\ldots$ & $>9$ & S & 17.03 \\
\hline $2010-10 b$ & $\ldots$ & 30 & $\ldots$ & $>41$ & $\ldots$ & 17.44 \\
\hline $2010-10 c$ & 1.0 & 3 & S & 20 & S & 16.65 \\
\hline $2010-10 \mathrm{e}$ & $\ldots$ & $\ldots$ & $\ldots$ & $>5$ & S & 17.84 \\
\hline $2010-12 a$ & 1.0 & 3 & S & $>13$ & $S$ & 15.56 \\
\hline $2010-12 b$ & $\ldots$ & $\ldots$ & $\ldots$ & 3 & $S$ & 15.70 \\
\hline
\end{tabular}

Note. ${ }^{\text {a }}$ The maximum magnitude is from ATel reports because PTF missed the peak magnitude.

This continuum UV light curve combined with the model of how the UV light is reprocessed into the optical light curve allows an estimate of the precursor WD mass and distance to be made, based on certain assumptions about the chemical composition of the envelope (Kato \& Hachisu 2007). 

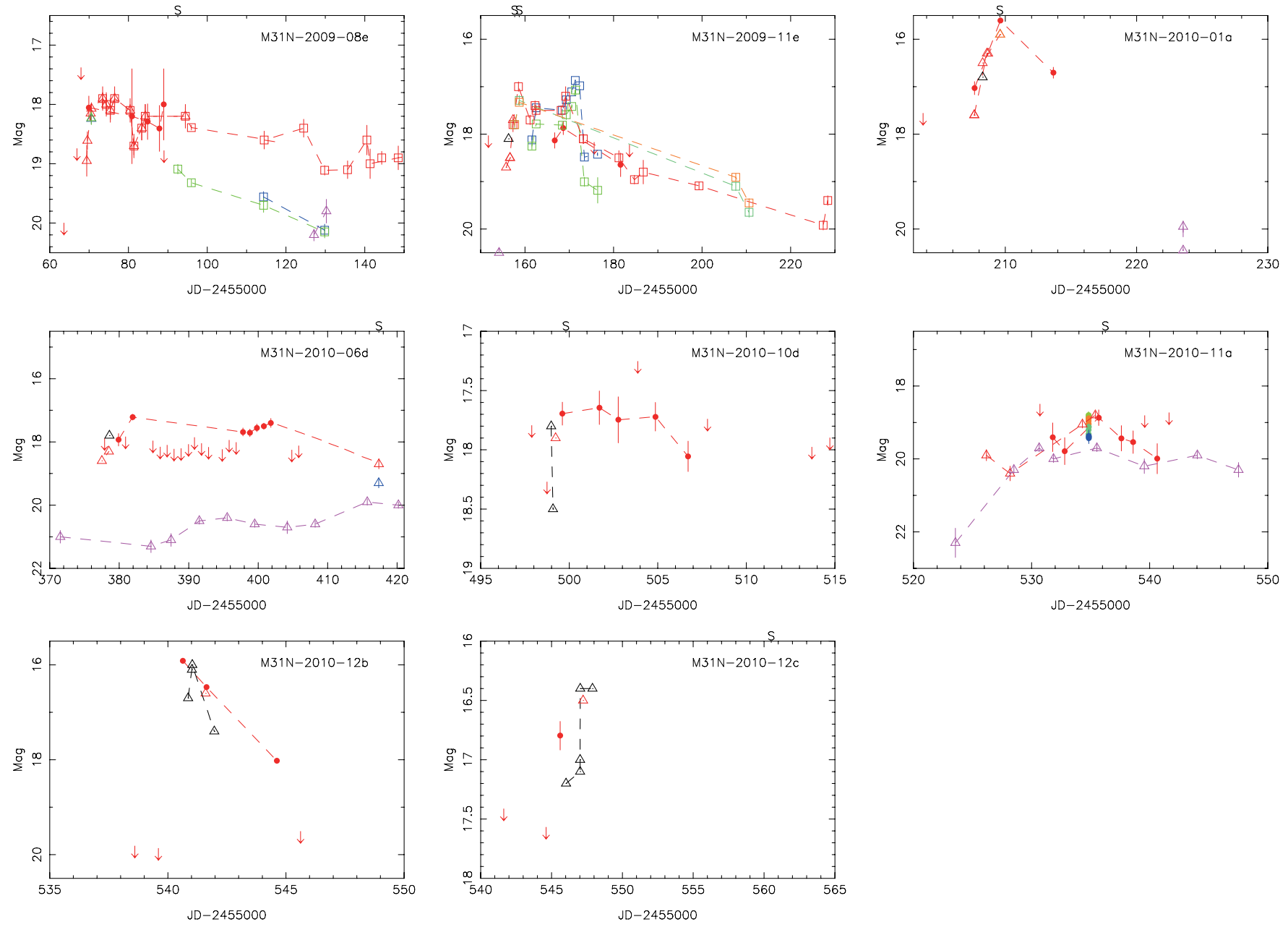

Figure 9. Light curves of undersampled novae. Symbol designations are the same as those in Figure 5.

(A color version of this figure is available in the online journal.)

One might assume that the clumpy wind responsible for the lower opacity and higher Eddington luminosities of the brightest novae might produce other observable signatures. Small-scale structure in the rising light curve could be observed as the clumps are non-uniformly heated and accelerated by the ongoing eruption and as radiation is diffused through them. We have very high cadence observations of the rising phases of two novae (M31N 2010-09a and M31N 2010-09c), both of which have peak brightnesses $\geqslant-7.5$ and are thus mildly super-Eddington novae (Duerbeck 1981). Nova 2010-09c exhibits a very smooth and steady rise and a smooth decline, consistent with a uniform nova wind, 2010-09a has considerable structure in its rising light curve with multiple peaks both near and after the maximum light. Our observations of M31N 2009-09a, M31N 2010-07a, M31N 2009-09a and M31N 2009-09c also show several peaks around maxima. The multiple peaks could arise when different winds are blown out from the center successively during an outburst. Each peak is formed when the pseudo-photosphere meets the shock wave at the head of the corresponding wind. These novae tend to be mildly to strongly super-Eddington; however, many of our novae with smooth, well-sampled light curves also fall into the range of mildly to strongly superEddington luminosity (e.g., M31N 2009-08b, but especially M31N 2010-09b). We therefore conclude that structures in the nova wind and thus in the light curve can arise regardless of the luminosity of the outburst.

\section{NOVAE IN GLOBULAR CLUSTERS}

M31N 2010-10f was spatially associated with the GC Bol 126 to within 1 arcsec. A supersoft X-ray source (SSS) was also reported in the vicinity (Henze et al. 2010d). This event is similar to M31N 2007-06b (Shafter \& Quimby 2007) in Bol 111 which was confirmed spectroscopically during the outburst. An SSS counterpart was also found for this object (Henze et al. 2009a). They are the only two events in M31 that are associated with GCs.

Several previous searches for GC novae obtained null results (e.g., Ciardullo et al. 1990b; Tomaney et al. 1992). We examined our PTF image database at the positions of all confirmed M31 GCs from the Revised Bologna Catalog (V.4, 2009 December; Galleti et al. 2004). Magnitudes of these GCs range from $R=13$ mag to $R=20 \mathrm{mag}$. We do not find any other GC novae candidates. Given an effective survey time of M31 in PTF for roughly one year, we obtain a GC nova rate of about $1 \mathrm{yr}^{-1}$.

Is the nova rate enhanced in GCs? The total stellar mass of M31 is $\sim 6 \times 10^{10} M_{\odot}$ (Tamm et al. 2007). Using the highest published nova rate for M31 of $65 \mathrm{yr}^{-1}$ from Darnley et al. (2006), we estimate a maximum global specific nova rate of $\sim 1 /\left(10^{9} M_{\odot}\right) \mathrm{yr}^{-1}$ in M31. Given a typical GC mass of $10^{6} M_{\odot}$ and about 700 confirmed M31 GCs, the nova rate in the M31 GC system is $\sim 1 /\left(10^{9} M_{\odot}\right) \mathrm{yr}^{-1}$. We conclude that the M31 

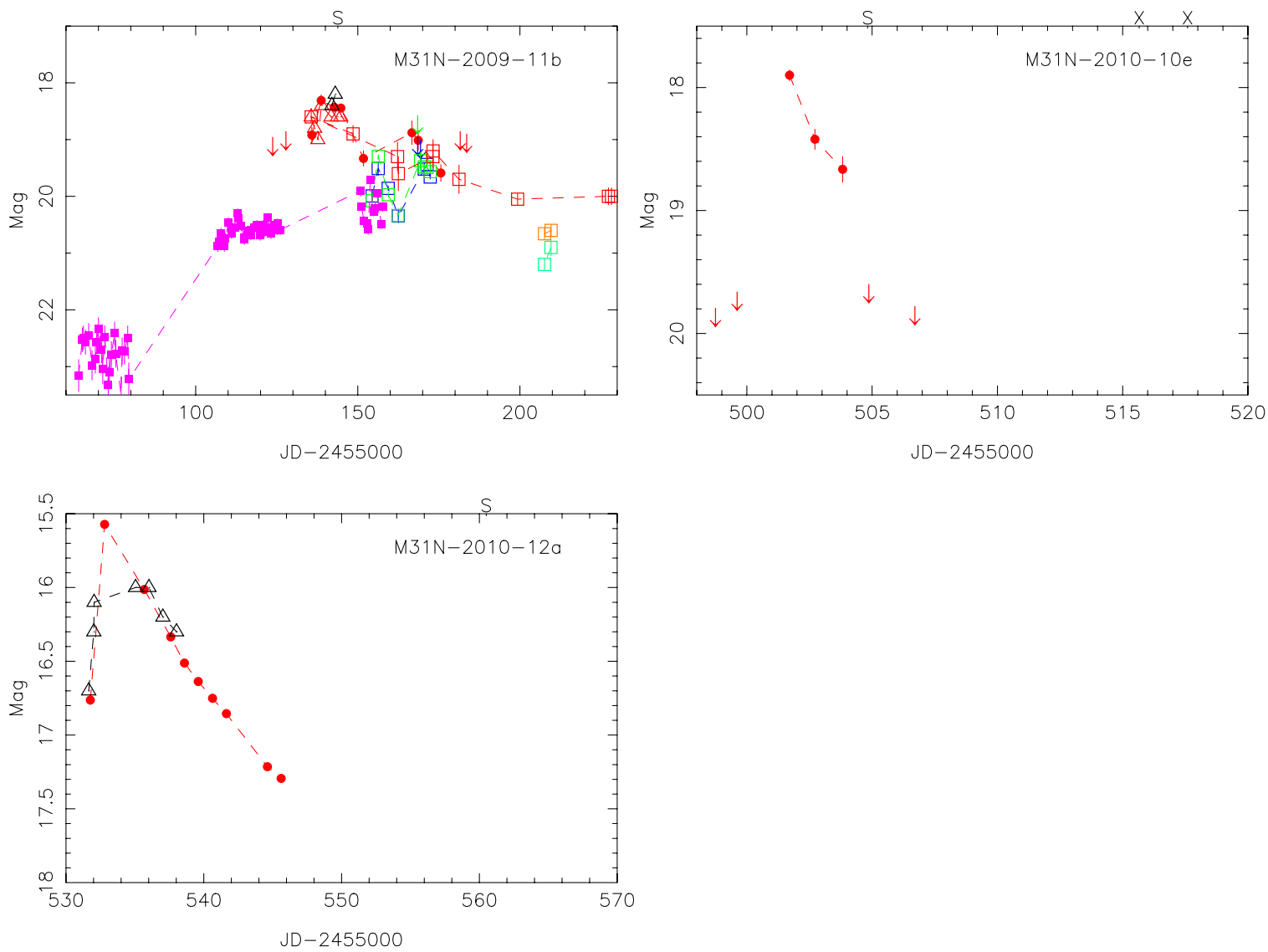

Figure 10. Light curves of three RNe detected in PTF. Symbol designations are the same as those in Figure 5.

(A color version of this figure is available in the online journal.)

GC specific nova rate is not significantly higher than the M31 galaxy specific nova rate.

Low-mass X-ray binaries (LMXBs) — mass transferring binaries with a neutron star receiving matter from a companion-can be viewed, in some ways, as surrogates for $\mathrm{CNe}$ and RNe. Since the early days of X-ray astronomy it has been known that, at least in our Galaxy, the rate of brightest LMXBs (per unit mass) are nearly two orders of magnitude larger than that for the disk of the Milky Way. This trend appears to be even more acute for M31 (Trudolyubov \& Priedhorsky 2004). This may be in part because M31 possesses a more extensive cluster system than our own Galaxy. Thus, naively, we would expect far more $\mathrm{CNe}$ and $\mathrm{RNe}$ in the GC system of M31. Over the period of investigation (2009-2010), a total of three RNe were found in the disk/bulge of M31. If we assume the mass fraction of GCs is $\sim 1 \%$, we would have expected to see a similar number in the cluster system. Within small number systems, the detection of an RN associated with the cluster would be consistent with this expectation. Separately, given that $700 \mathrm{CNe}$ are known, over 2009 and 2010 we should have seen many tens of CNe in the GCs. but only one was found. Indeed, over the last hundred years only one CN that has been associated with a GC of M31 has been spectroscopically confirmed (Shafter \& Quimby 2007). In summary, we cannot arrive at a sensible conclusion with the presently available data.

\section{RECURRENT NOVAE}

According to the catalog maintained by Pietsch (2010), 26 $\mathrm{RNe}$ in M31 are known. During the two seasons we report on, three recurrent events were reported: M31N 2009-11b, M31N 2010-10e, and M31N 2010-12a. Figure 10 presents their light curves. Besides these, we examined the PTF archive by putting an aperture at each position of more than 800 old novae in M31 from the list of Pietsch (2010) in order to find more recurrent events. None were found. Therefore, given an effective observing time of roughly one year in PTF, the RN rate in M31 is $\sim 3$ novae per year. Given a total number of 29 novae in our sample, we derived an outburst ratio of $\mathrm{RNe}$ and $\mathrm{CNe}$ to be about 0.1 , consistent with that of della Valle \& Livio (1996).

We examined M31N 2010-12a in some detail, as another outburst of PT And. This object has recorded outbursts in 1957, 1983, 1986, 1988, and 1998. Alksnis \& Zharova (2000) collected its previous outbursts and found that all light curves can be fit by one template. The average decline rate of previous outbursts is $0.09-0.11 \mathrm{mag} \mathrm{day}^{-1}$. The light curve of this most recent outburst (M31N 2010-12a) can also be fit by the template. Figure 11 shows the similarity between light curves of the recent and 1988 outbursts. The decline rate of the recent outburst is around $0.10 \mathrm{mag} \mathrm{day}^{-1}$ consistent with previous ones. This invariance property is also observed in Galactic RNe (Schaefer 2010). Such characteristics of RNe suggests that RN outbursts only depend on system parameters such as the WD mass and its composition.

Because of its luminous and frequent outbursts, PT And was assumed to be a dwarf nova inside the Milky Way (Alksnis \& Zharova 2000). However, no direct evidence of this object's nature was available until we obtained a spectrum of its most recent outburst with LRIS in the Keck I telescope. This spectrum (see the top panel of Figure 3) shows a very blue continuum 


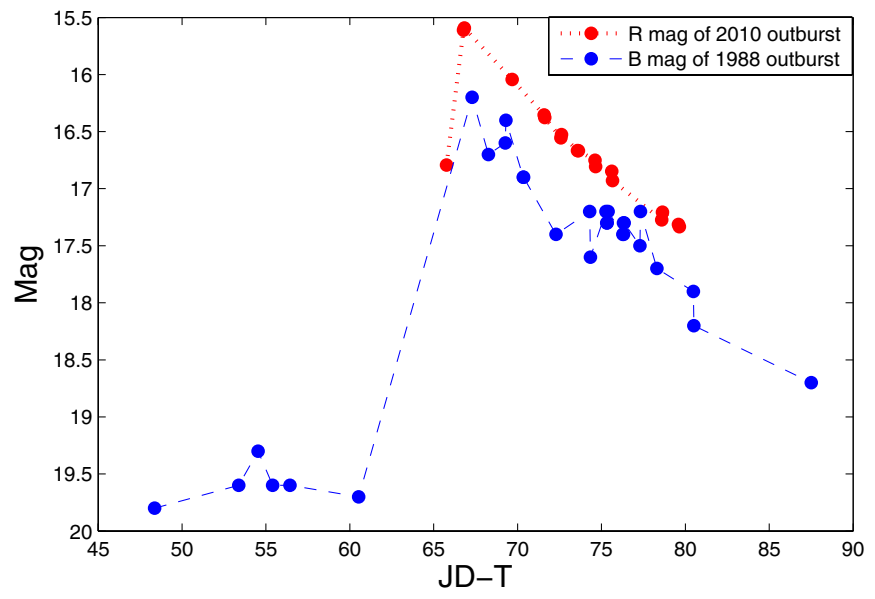

Figure 11. Comparison of the light curves of outbursts of PT And in 1988 and 2010 is shown in the figure, where $T=2455,466$ for the 2010 outburst and $T=2455,100$ for the 1988 one. Though with different filters, the light curve of the recent outburst (M31N 2010-12a) resembles that of the 1988 outburst.

(A color version of this figure is available in the online journal.)

superposed with prominent $\mathrm{H} \alpha$ and $\mathrm{H} \beta$ lines as well as several weak Fe lines. Fitting the red part of the spectrum with a Planck function yields a temperature of $7 \sim 8 \times 10^{3} \mathrm{~K}$. We fit the $\mathrm{H} \alpha$ and $\mathrm{H} \beta$ lines with Gaussian profiles and obtained the following observed wavelengths: $\mathrm{H} \alpha$ is centered at $\lambda=6558 \AA$ and $\mathrm{H} \beta$ at $\lambda=4857 \AA$. These correspond to blueshifts with radial velocities of $\sim-200 \mathrm{~km} \mathrm{~s}^{-1}$ and $\sim-300 \mathrm{~km} \mathrm{~s}^{-1}$. The systemic velocity of M31 is $\sim-300 \mathrm{~km} \mathrm{~s}^{-1}$. We assume that this object is not located in the disk of M31, thus we do not take the rotation velocity of M31 disk into account. We conclude that the blueshifts are roughly consistent with the motion of M31. Our spectrum evidently supports the conclusion that PT And resides in M31 instead of in the Milky Way. This can also be tested by observing its proper motion. It is of only galactic sources that we may observe proper motion. The FWZI of the Balmer lines suggest an expansion velocity of $\simeq 2000 \mathrm{~km} \mathrm{~s}^{-1}$. In addition, given very weak iron lines, we tentatively classify the nova into the Fe II type.

The association of PT And with M31 leads to a peak absolute magnitude of about -9 , which makes it the brightest $\mathrm{RN}$ ever known.

The decline rates of RNe vary over a large range in M31. M31N 2009-11b has $t_{2}>37$ days and M31N 2010-10e has $t_{2}>5$ days. Consider that M31N 2010-12a has a decline rate of $\sim 0.1 \mathrm{mag} \mathrm{day}^{-1}$, so its $t_{2}$ is possibly around 20 days. The $t_{2}$ values of Galactic $\mathrm{RNe}$ also range from 1 day to 50 days (Schaefer 2010).

\section{UV LIGHT CURVES OF NOVAE}

Theoretically, the UV emission after the optical maximum light is characterized by a delayed UV peak after the optical peak, because most UV photons are absorbed and re-emitted in the optical in the outer envelope (Kato \& Hachisu 1994; González-Riestra \& Krautter 1998; Cassatella et al. 2002). However in our sample, we see some deviation from this general picture: $2009-10$ b has a peak in the GALEX NUV over 2 days prior to its peak in the optical (see Figure 12). The RN 2009$11 \mathrm{~b}$ was seen in the GALEX NUV about 40 days before it was visible in optical bands (although this could be due to cadence and weather, see Figure 10). The nova 2010-06d was detected in Swift (Pietsch et al. 2010f) about 6 days before optical detection

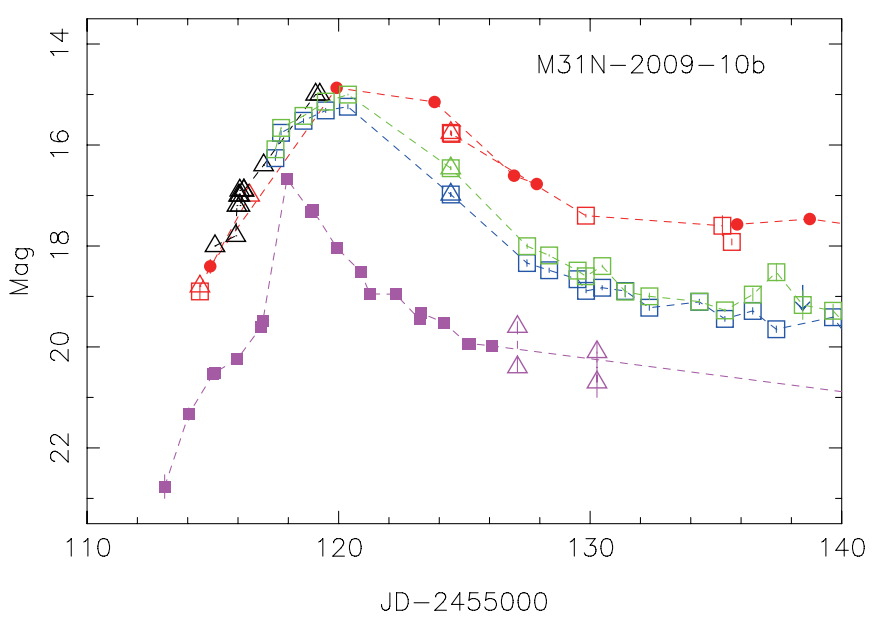

Figure 12. The multi-band light curve of M31 N2009-10b focusing on its early evolution. We can clearly see that the UV peaks prior to the peaks in $B, V$, and $R$ bands by about 2 days. When the UV decays, the optical part is quite red. Symbol designations are the same as those in Figure 5.

(A color version of this figure is available in the online journal.)

(see Figure 9). Swift also detected 2010-11a several days before optical detection (Pietsch et al. 2010h; Cao et al. 2010; also Figure 9). For 2010-07a, the optical and UV maxima were nearly simultaneous as measured by PTF and by Swift (Henze et al. 2010b; see Figure 7).

Admittedly the detection in the optical and UV involves many factors including survey sensitivities, temporal coverage, and observing weather. In a few cases, however, it is clear that the UV peak coincides with or precedes the optical peak. These cases demonstrate that not all UV photons are re-processed and that the absorption in the UV will vary considerably depending on factors such as physical geometry or chemical abundances in the envelope. These variations will produce a range of optical/ UV peak offsets and luminosity ratios. We also point out that multi-band optical data show that three of these UV novae have red colors: 2009-10b has $R-V \sim 1.4$ at decline (Shafter et al. 2011b), 2009-11b has $R-V \sim 0.7$ at decline (Shafter et al. 2011b), and 2010-11a has $g-i \sim 0.2$ around its optical peak from our photometric follow-up.

\subsection{M31N 2009-10b and M31N 2010-11a}

The novae 2009-10b and 2010-11a are two peculiar cases in that their UV peaks lead the optical maxima by a few days. Moreover, at the early decay stage of UV emission, the optical colors of both novae look quite red.

Of the two, 2009-10b is brighter, has better light curve coverage in PTF, GALEX, and other bands, and is thus a better case for further examination. Figure 12 zooms in on the early multi-band light curve. Table 7 lists the rise rate, maximum magnitudes, dates, and decline rates by $t_{2}$ and $t_{3}$ in the NUV, $B, V$, and $R$ bands. The NUV data are from GALEX. Part of the $R$-band data are from PTF. $B, V$ and part of $R$-band data are published in Shafter et al. (2011b). We can see clearly that the NUV peak is two-to-three days earlier than the $B, V$, and $R$ peaks. The rise rates in the $B, V$, and $R$ bands are consistent while the NUV emission rises swiftly. The decline rates of NUV, $B$, and $V$ are similar while the $R$-band emission decays slower.

2010-11a is possibly the faintest nova in our sample with a peak-observed magnitude of $R \sim 18.8$ (see Figure 9). It was first detected by Swift (Pietsch et al. 2010h) and then we reported its optical counterpart (Cao et al. 2010). The strong UV emission 
Table 7

2009-10b NUV and Optical Properties

\begin{tabular}{lcccc}
\hline \hline Bands & NUV $^{\mathrm{a}}$ & $B^{\mathrm{b}}$ & $V^{\mathrm{b}}$ & $R^{\mathrm{c}}$ \\
\hline Rise rate (mag day & \\
Peak magnitude & 1.2 & 0.4 & 0.4 & 0.7 \\
Peak date (JD-2455110) & 16.68 & 15.24 & 15.00 & 14.84 \\
$t_{2}$ (days) & 7.95 & 10.37 & 10.37 & 9.95 \\
$t_{3}$ (days) & 3 & 5 & 5 & 8 \\
\end{tabular}

Notes.

a GALEX observations.

b Data from Shafter et al. (2011b).

${ }^{c}$ Data from PTF and Shafter et al. (2011b).

Table 8

P60 Photometric Follow-up of M31N 2010-11a

\begin{tabular}{llcc}
\hline \hline $\begin{array}{l}\text { JD-2455500 } \\
\text { (days) }\end{array}$ & Bands & Magnitude & Magnitude Error \\
\hline 34.817 & $i^{\prime}$ & 18.80 & 0.10 \\
34.819 & $i^{\prime}$ & 18.84 & 0.09 \\
34.822 & $i^{\prime}$ & 18.89 & 0.09 \\
34.824 & $r^{\prime}$ & 18.91 & 0.08 \\
34.826 & $r^{\prime}$ & 19.04 & 0.09 \\
34.838 & $r^{\prime}$ & 18.96 & 0.08 \\
34.831 & $r^{\prime}$ & 18.89 & 0.09 \\
34.833 & $r^{\prime}$ & 19.04 & 0.11 \\
34.835 & $g^{\prime}$ & 19.20 & 0.09 \\
34.838 & $g^{\prime}$ & 19.32 & 0.11 \\
34.840 & $g^{\prime}$ & 19.12 & 0.09 \\
34.842 & $B$ & 19.40 & 0.13 \\
34.844 & $B$ & 19.37 & 0.16 \\
34.847 & $B$ & 19.43 & 0.15 \\
\hline
\end{tabular}

peaked several days prior to its optical maximum. Near its optical maximum, we carried out a photometric follow-up in the $i^{\prime}, r^{\prime}, g^{\prime}$, and $B$ bands with the Palomar 60 inch telescope and observed that this nova had red optical colors, as shown in Table 8.

The UV observations and the color information suggest that UV and optical emission originates from different radiative regions because they cannot be simply interpreted as the thermal radiation of a receding spherical photosphere which has a large optical depth in local thermal equilibrium. Because UV emission is only generated deep inside the envelope, the result may suggest that the envelope has holes from which we can see deep inside.

The models of Shaviv (2001) and Kato \& Hachisu (2005, 2007) propose a reduced effective opacity of the nova envelope to explain the super-Eddington phase, resulting from a porous structure of the envelope caused by flow instabilities. We may use a similar scenario of asphericity of nova outbursts to qualitatively explain the red UV novae. UV photons are generated deep under the envelope at the surface of the WD. In the envelope, the bottom is heated to very high temperatures by thermonuclear runaway, and thus instabilities like Rayleigh-Bénard convection are likely to occur. This instability causes the mass distribution in the nova envelope to deviate from a spherical distribution. Thus, along some lines of sight to the center of the outburst, the mass is less and so is the opacity. The UV photons then escape from these directions and are observed by us if we are observing along one of these lines of sight. The red optical emission still comes from the reprocessing of the envelope in the other directions. The ratio between the UV and optical fluxes reflects the

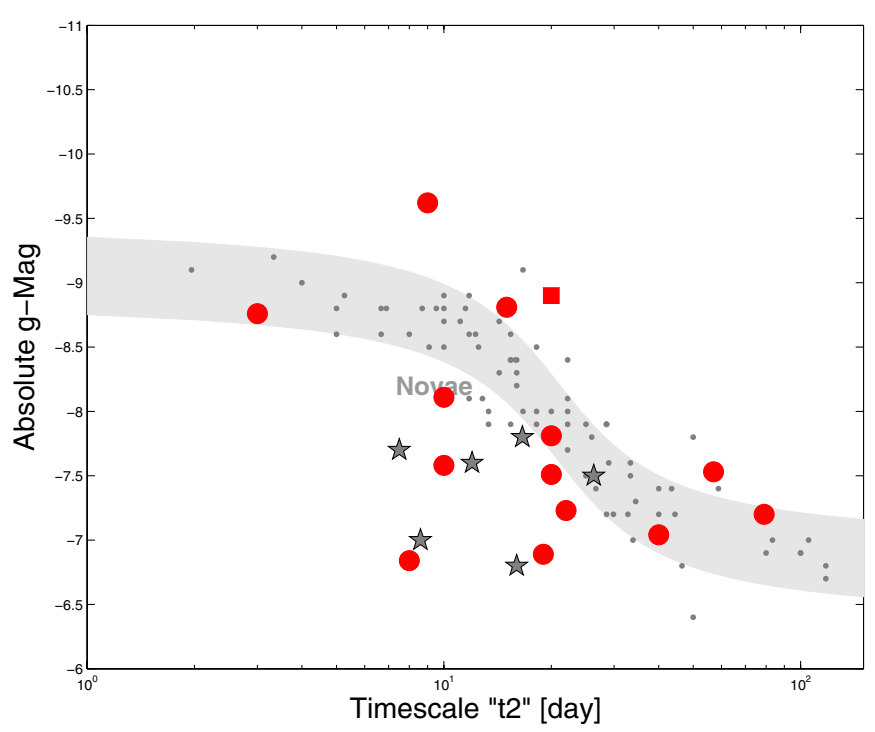

Figure 13. Empirical relation for novae between the maximum magnitude and the rate of decline (MMRD). The gray region denotes the MMRD from della Valle \& Livio (1995), while the small dark gray solid dots represent their nova sample. The six gray stars show the sample from Kasliwal et al. (2011). Red circles and a red square are the M31 novae from this paper. The red square denotes the bright recurrent nova M31N 2010-12a.

(A color version of this figure is available in the online journal.)

angular size of the cone. If the instability produces an extreme feature with very low mass and very low opacity along the line of sight, we may detect the central UV photons directly. This might be what we are observing in 2009-10b and 2010-11a. Another possible explanation could be very low abundances of chemical species that absorb in the UV, although this may not explain the red optical colors.

The uniqueness of the UV-optical light curves of 2009-10b and 2010-11a suggest that either their super-Eddington phases or their chemical abundances (or both) are very different from the majority of novae (Cassatella et al. 2002). They may possibly represent a population that deviates from any empirical relation derived from large nova samples, such as MMRD relation. In fact, 2009-10b is a luminous outlier of the MMRD relation (see Figure 13). Unfortunately, due to its faintness, we were unable to measure the $t_{2}$ decline rate for the nova 2010-11a.

\section{MMRD REVISITED}

Novae exhibit an MMRD relation (della Valle \& Livio 1995; Kasliwal et al. 2011). We can assess this relation with our nova sample in M31.

One of the main issues in evaluating the MMRD relation is estimating the dust extinction. A multitude of methods using color and Balmer decrement have been used in literature to estimate the extinction (Darnley et al. 2006; Kogure 1961; Kasliwal et al. 2011). The PTF nova observations, however, provide no color information, being done only in the $R$ band. Thus, we apply a mean foreground dust extinction. The foreground extinction of M31, $E(B-V)=0.062$ mag (Schlegel et al. 1998), leads to

$$
A_{g}=3.793 \times E(B-V)=0.234 .
$$

For a better comparison with previous results (e.g., Kasliwal et al. 2011), following Shafter et al. (2009), we use the colors of an A5V star $(T=8200 \mathrm{~K})$ to transform $R$ magnitudes to $g$ magnitudes (Jordi et al. 2006). 
The MMRD relation is presented as the gray band in Figure 13. We overplot on this diagram our M31 novae (red points) and compare them to the novae from della Valle \& Livio (1995, gray points) and Kasliwal et al. (2011, gray stars). Without a local extinction correction, most of our peak magnitudes are underestimated. In Kasliwal et al. (2011), the total extinction correction for M31 novae is roughly $A_{g}=0.24$ except that two of them (20\%) have a correction of $A_{g}>1.0$. It is possible that local extinction for most novae in our sample is small.

In Figure 13, we can see five significant outliers from the MMRD region in our sample. The brightest outlier is M31N 2010-10b (see discussion in Section 6.1). The other luminous outlier is PT And (=M31N 2010-12a). M31N 2009$12 \mathrm{a}, \mathrm{M} 31 \mathrm{~N} 2010-06 \mathrm{~b}$, and M31N 2010-06c lie in the faint and fast-decline rate zone below the MMRD relation shown by the gray band. Kasliwal et al. (2011) also found several novae in this zone. Kasliwal et al. (2011) proposed the possibility that the outliers may be RNe. However, none of the three faint outliers in our sample are known to be recurrent. The remaining novae reside roughly along the MMRD region.

M31N 2010-10b resides near the brightest end of novae, which resembles "superbright" novae that have been observed in M31, the Large Magellanic Cloud, and the Galaxy (e.g., della Valle 1991). The energy budget for these objects is not clear. It could be related to the common envelope phase in nova systems (e.g., Iben \& Livio 1993) or a very cold low-mass WD accreting through Roche lobe from a close low-mass companion (Iben \& Tutukov 1992). In either scenario, as the outer part of the system comes from the companion, the UV observation of M31N 2010$10 \mathrm{~b}$ requires very low metallicity of the common envelope of the binary system or the outer envelope of the nova. Therefore, correlations between spatial distributions of stellar population and "superbright" novae will be helpful.

\section{CONCLUSION}

In this paper, we tabulate the photometric measurements of $29 \mathrm{CNe}$ in M31 from PTF data and present their optical light curves, eight of which also have joint UV-optical light curves from both PTF and GALEX. The main findings are as follows.

1. We detected three RNe. They show similar properties to $\mathrm{RNe}$ in the Galaxy (Schaefer 2010). We obtained a spectrum of the recent outburst of PT And (also known as M31N 2010-12a). While previously identified as a dwarf nova in the Milky Way (Alksnis \& Zharova 2000), we find the blueshifts in our spectrum to be consistent with the event being an RN in M31. The light curve of 2010-12a resembles those of previous outbursts, suggesting the same physical conditions in the recurrent outbursts. Our search at all other historical nova positions finds no additional outburst candidates. From this we derive an RN rate of about three per year in M31.

2. The rise behaviors of $\mathrm{M} 31 \mathrm{CNe}$ are diverse and we classify them morphologically into S (smooth) and J (jittering) classes. We find that the S-class novae rise significantly faster than the J-class novae. We see no dependence between rise time and maximum magnitude.

3. During the 10 minute cadence PTF observations the rising light curve of two novae were recorded with unprecedented detail. These novae showed smooth and consistent rising light curves without large amplitude variability.
4. The declining light curves are also divided morphologically into $\mathrm{S}$ and $\mathrm{J}$ classes. The relative population of different classes in M31 is roughly consistent with that in the Galaxy.

5. Three quarters of the well-sampled novae obey the empirical MMRD relation. The remaining one quarter (five events) are consistent with the "faint and fast" outliers found by Kasliwal et al. (2011).

6. Some novae have UV detections prior to or at the same time as the first optical detection. Moreover, 2009-10b and 2010-11a have UV peaks prior to optical maxima while both are quite red in the UV decay phases. These observations are inconsistent with theoretical predictions of delayed UV emission in the iron curtain stage, possibly suggesting extreme density or abundance variations for these novae.

7. Apart from 2010-10f in Bol 126, we find no other novae in all the cataloged M31 GCs. We derive a GC nova rate in M31 of $\sim 1 \mathrm{yr}^{-1}$. This nova rate is not enhanced relative to the rate in M31 outside of the GC system.

We realize the novae reported here are only a subset of all the $\mathrm{CNe}$ in $\mathrm{M} 31$ as the number is smaller than expected (e.g., 65 nova $\mathrm{yr}^{-1}$ according to Darnley et al. 2006). We did not undertake a systematic search for $\mathrm{CNe}$ in PTF images during the 2009 and 2010 seasons. Since the FoV and survey depth of PTF should be sensitive to all CNe (weather-permitting), we have started a more complete search for CNe in M31 in the 2011 season. For 2012, we intend to run a real-time search for novae. Our photometric and spectroscopic follow-up will enable us to better understand their properties and to estimate the local extinction correction.

Besides optical bands, future UV surveys are warranted for nova studies. Some novae peak in the UV before the optical. Such novae could provide important insights into the development and structure of the nova wind. It is also important to know if novae such as M31N 2009-10b and M31N 2010-11a are rare or common and if they are always outliers in the MMRD diagram.

Ongoing and upcoming synoptic surveys (e.g., PTF and Next Generation Transient Facilities) will continue to monitor the Andromeda Galaxy. The long time baseline will give us a larger sample of RNe. As noted earlier, RNe could be the progenitors of Ia supernovae. Thus, the increased sample and timely follow-up will give us a comprehensive understanding of rate of recurrent novae-an important clue to their endpoint.

We thank the referee, Dr. Massimo Della Valle, for very helpful comments and suggestions to improve the manuscript. We thank Marina Orio and Sumin Tang for valuable discussions. We thank the Weizmann Monitoring Team (A. Gal-Yam, I. Arcavi, D. Polishook, A. Sternberg, O. Yaron, D. Xu) for daily monitoring of transient candidates from the PTF discovery stream.

M.M.K. acknowledges support from the Hubble Fellowship and the Carnegie-Princeton Fellowship. S.B.C wishes to acknowledge generous support from Gary and Cynthia Bengier, the Richard and Rhoda Goldman Fund, National Aeronautics and Space Administration (NASA)/Swift grant NNX10AI21G, NASA/Fermi grant NNX1OA057G, and National Science Foundation (NSF) grant AST-0908886.

This research was supported in part by Tsinghua Center for Astrophysics (THCA), by the National Natural Science Foundation of China (NSFC) grants 10373009, 10533020, and 11073014 at Tsinghua University, by the Ministry of Science 
and Technology (MOST) under State Key Development Program for Basic Research grant 2012CB821800, by the Tsinghua University Initiative Scientific Research Program, by the SRFDP 20050003088, 200800030071, and 20110002110008, and by the Yangtze Endowment from the Ministry of Education at Tsinghua University.

GALEX (Galaxy Evolution Explorer) is a NASA Small Explorer, launched in 2003 April. We gratefully acknowledge NASA's support for construction, operation, and science analysis for the GALEX mission, developed in cooperation with the Centre National d'Etudes Spatiales of France and the Korean Ministry of Science and Technology.

The National Energy Research Scientific Computing Center, which is supported by the Office of Science of the U.S. Department of Energy under Contract No. DE-AC02-05CH11231, provided staff, computational resources and data storage for PTF.

This research has made use of the NASA/IPAC Extragalactic Database (NED) which is operated by the Jet Propulsion Laboratory, California Institute of Technology, under contract with the National Aeronautics and Space Administration.

\section{REFERENCES}

Alksnis, A., \& Zharova, A. V. 2000, IBVS, 4909, 1

Arp, H. C. 1956, AJ, 61, 15

Barsukova, E., Afanasiev, V., Fabrika, S., et al. 2009a, ATel, 2251, 1

Barsukova, E., Valeev, A., Sholukhova, O., et al. 2009b, ATel, 2205, 1

Barsukova, E. A., Valeev, A. F., Sholukhova, O., et al. 2010, ATel, 2789, 1

Bode, M., \& Evans, A. 2008, Classical Novae (Cambridge Astrophysics Series; Cambridge: Cambridge Univ. Press)

Burwitz, V., Pietsch, W., Henze, M., et al. 2010a, CBET, 2343, 1

Burwitz, V., Pietsch, W., Henze, M., et al. 2010b, ATel, 2697, 1

Burwitz, V., Pietsch, W., Henze, M., et al. 2010c, CBET, 2124, 1

Burwitz, V., Pietsch, W., Henze, M., et al. 2010d, ATel, 2383, 1

Burwitz, V., Rodriguez, J., Holmes, S., Kolb, U., \& Lucas, R. 2009, CBET, 2003, 2

Cagas, P. 2009, CBET, 2061, 3

Cao, Y., Kasliwal, M. M., \& Kulkarni, S. R. 2010, ATel, 3066, 1

Cassatella, A., Altamore, A., \& González-Riestra, R. 2002, A\&A, 384, 1023

Cassatella, A., Lamers, H. J. G. L. M., Rossi, C., Altamore, A., \& GonzálezRiestra, R. 2004, A\&A, 420, 571

Cenko, S. B., Fox, D. B., Moon, D.-S., et al. 2006, PASP, 118, 1396

Chemin, L., Carignan, C., \& Foster, T. 2009, ApJ, 705, 1395

Ciardullo, R., Ford, H. C., Neill, J. D., Jacoby, G. H., \& Shafter, A. W. 1987, ApJ, 318,520

Ciardullo, R., Shafter, A. W., Ford, H. C., et al. 1990a, ApJ, 356, 472

Ciardullo, R., Tamblyn, P., \& Phillips, A. C. 1990b, PASP, 102, 1113

Corbelli, E., Lorenzoni, S., Walterbos, R., Braun, R., \& Thilker, D. 2010, A\&A, 511, A89

Corral-Santana, J., Casares, J., Hornochova, P., \& Wolf, M. 2010, CBET, 2487, 1

Darnley, M. J., Bode, M. F., Kerins, E., et al. 2004, MNRAS, 353, 571

Darnley, M. J., Bode, M. F., Kerins, E., et al. 2006, MNRAS, 369, 257

della Valle, M. 1991, A\&A, 252, L9

della Valle, M., \& Livio, M. 1995, ApJ, 452, 704

della Valle, M., \& Livio, M. 1996, ApJ, 473, 240

Di Mille, F., Ciroi, S., Navasardyan, H., et al. 2009, ATel, 2248, 1

Duerbeck, H. W. 1981, PASP, 93, 165

Fabrika, S., Barsukova, E. A., Valeev, A. F., et al. 2010, ATel, 3068, 1

Fabrika, S., Sholukhova, O., Valeev, A., et al. 2009a, CBET, 1971, 3

Fabrika, S., Sholukhova, O., Valeev, A., Hornoch, K., \& Pietsch, W. 2009b, ATel, 2239, 1

Galleti, S., Federici, L., Bellazzini, M., Fusi Pecci, F., \& Macrina, S. 2004, A\&A, 416, 917

Gehrels, N., Chincarini, G., Giommi, P., et al. 2004, ApJ, 611, 1005

Gil de Paz, A., Boissier, S., Madore, B. F., et al. 2007, ApJS, 173, 185

González-Riestra, R., \& Krautter, J. 1998, in Ultraviolet Astrophysics Beyond the IUE Final Archive, ed. W. Wamsteker, R. Gonzalez Riestra, \& B. Harris (ESA Special Publication, Vol. 413; Noordwijk, The Netherlands: ESA), 367 Green, D. W. E. 2009, CBET, 2061, 1

Green, D. W. E. 2010a, CBET, 2582, 1
Green, D. W. E. 2010b, CBET, 2582, 4

Green, D. W. E. 2010c, CBET, 2582, 5

Green, D. W. E. 2010d, CBET, 2597, 2

Green, D. W. E. 2010e, CBET, 2187, 1

Green, D. W. E. 2010f, CBET, 2610, 1

Henze, M., Burwitz, V., Pietsch, W., et al. 2010a, CBET, 2358, 2

Henze, M., Burwitz, V., Pietsch, W., et al. 2010b, ATel, 2727, 1

Henze, M., Haberl, F., Sala, G., et al. 2009a, A\&A, 500, 769

Henze, M., Kaduk, F., Pietsch, W., et al. 2009b, ATel, 2189, 1

Henze, M., Pietsch, W., \& Haberl, F. 2010c, ATel, 2787, 1

Henze, M., Pietsch, W., Haberl, F., \& Orio, M. 2009c, ATel, 2274, 1

Henze, M., Pietsch, W., Podigachoski, P., et al. 2009d, ATel, 2286, 1

Henze, M., Pietsch, W., Podigachoski, P., et al. 2009e, CBET, 2015, 2

Henze, M., Pietsch, W., Burwitz, V, et al. 2010d, ATel, 3019, 1

Hornoch, K. 2010, CBET, 2343, 3

Hornoch, K., Corral-Santana, J. M., \& Casares, J. 2010a, CBET, 2391, 1

Hornoch, K., Corral-Santana, M., Casares, J., et al. 2010b, CBET, 2411, 1

Hornoch, K., Hornochova, P., Gallagher, J., \& Garnavich, P. 2010c, CBET, 2342, 1

Hornoch, K., Hornochova, P., Kubanek, P., et al. 2010d, CBET, 2341, 1 Hornoch, K., Hornochova, P., Kubanek, P., et al. 2010e, CBET, 2341, 2 Hornoch, K., Hornochova, P., \& Wolf, M. 2010f, CBET, 2516, 4

Hornoch, K., Hornochova, P., Zasche, P., \& Wolf, M. 2010g, CBET, 2391, 3

Hornoch, K., Kubanek, P., Gorosabel, J., et al. 2010h, CBET, 2187, 3

Hornoch, K., \& Kusnirak, P. 2009, CBET, 1971, 2

Hornoch, K., Kusnirak, P., \& Pejcha, O. 2009a, CBET, 1971, 4

Hornoch, K., \& Pejcha, O. 2009, CBET, 2061, 5

Hornoch, K., Pejcha, O., \& Kusnirak, P. 2009b, CBET, 2058, 3

Hornoch, K., Pejcha, O., \& Wolf, M. 2009c, CBET, 2062, 1

Hornoch, K., Prieto, J. L., Khan, R., \& Pejcha, O. 2010i, CBET, 2136, 1

Hornoch, K., Prieto, J. L., Khan, R., et al. 2010j, CBET, 2127, 1

Hornoch, K., Prieto, J. L., Pejcha, O., et al. 2010k, CBET, 2391, 2

Hornoch, K., Wolf, M., Garnavich, P., et al. 20101, CBET, 2347, 2

Hornoch, K., \& Zasche, P. 2010, CBET, 2358, 1

Hornochova, P., Hornoch, K., Wolf, M., Kusnirak, P., \& Pejcha, O. 2010, CBET, 2573,1

Hornochova, P., \& Wolf, M. 2010, CBET, 2571, 1

Hubble, E. P. 1929, ApJ, 69, 103

Iben, I., Jr., \& Livio, M. 1993, PASP, 105, 1373

Iben, I., Jr., \& Tutukov, A. V. 1992, ApJ, 389, 369

Jordi, K., Grebel, E. K., \& Ammon, K. 2006, A\&A, 460, 339

Kasliwal, M. M. 2009, CBET, 2015, 3

Kasliwal, M. M., Cenko, S. B., Kulkarni, S. R., et al. 2011, ApJ, 735, 94

Kasliwal, M. M., Quimby, R., Shara, M., et al. 2009, ATel, 2290, 1

Kato, M., \& Hachisu, I. 1994, ApJ, 437, 802

Kato, M., \& Hachisu, I. 2005, ApJ, 633, L117

Kato, M., \& Hachisu, I. 2007, ApJ, 657, 1004

Kogure, T. 1961, PASJ, 13, 335

Koishikawa, M. 2010, CBET, 2582, 2

Koishikawa, M., Pietsch, W., \& Yusa, T. 2010, CBET, 2582, 7

Kron, R. G. 1980, ApJS, 43, 305

Krushinski, V., Zalozhnich, I., Kopytova, T., \& Popov, A. 2010, ATel, 2844, 1

Kusnirak, P., Hornoch, K., Zasche, P., \& Wolf, M. 2009, CBET, 2061, 4

Law, N. M., Kulkarni, S. R., Dekany, R. G., et al. 2009, PASP, 121, 1395

Martignoni, M., Itagaki, K., \& Yamaoka, H. 2009, CBET, 1980, 2

Martin, D. C., Fanson, J., Schiminovich, D., et al. 2005, ApJ, 619, L1

Massey, P., Olsen, K. A. G., Hodge, P. W., et al. 2006, AJ, 131, 2478

Medvedev, A., Barsukova, E., Valeev, A., et al. 2009, ATel, 2213, 1

Merrett, H. R., Merrifield, M. R., Douglas, N. G., et al. 2006, MNRAS, 369, 120

Morrissey, P., Conrow, T., Barlow, T. A., et al. 2007, ApJS, 173, 682

Nakano, S., \& Itagaki, K. 2009, CBET, 1973, 1

Nakano, S., Itagaki, K., \& Nicolas, J. 2009, CBET, 1971, 5

Nakano, S., \& Yusa, T. 2009, CBET, 1967, 1

Nakano, S., \& Yusa, T. 2010, CBET, 2500, 1

Nishiyama, K., \& Kabashima, F. 2009a, CBET, 2003, 1

Nishiyama, K., \& Kabashima, F. 2009b, CBET, 2058, 1

Nishiyama, K., \& Kabashima, F. 2010, CBET, 2516, 2

Nishiyama, K., Kabashima, F., Hornoch, K., et al. 2009a, CBET, 2015, 1

Nishiyama, K., Kabashima, F., \& Yusa, T. 2009b, CBET, 2100, 1

Oke, J. B., Cohen, J. G., Carr, M., et al. 1995, PASP, 107, 375

Ovcharov, E., Valcheva, A., Georgiev, T., et al. 2009, ATel, 2176, 1

Pietsch, W. 2010, Astron. Nachr., 331, 187

Pietsch, W., \& Henze, M. 2010a, CBET, 2187, 2

Pietsch, W., \& Henze, M. 2010b, ATel, 2435, 1

Pietsch, W., Henze, M., Burwitz, V., et al. 2010a, CBET, 2582, 3

Pietsch, W., Henze, M., Burwitz, V., et al. 2010b, ATel, 3001, 1 
Pietsch, W., Henze, M., Burwitz, V., et al. 2010c, ATel, 3076, 1 Pietsch, W., Henze, M., Burwitz, V., et al. 2010d, CBET, 2343, 2 Pietsch, W., Henze, M., Burwitz, V., et al. 2010e, CBET, 2347, 1

Pietsch, W., Henze, M., Burwitz, V., et al. 2010f, ATel, 2713, 1

Pietsch, W., Henze, M., Haberl, F., \& Burwitz, V. 2010g, ATel, 3038, 1

Pietsch, W., Henze, M., Haberl, F., \& Burwitz, V. 2010h, ATel, 3061, 1

Pietsch, W., Henze, M., Haberl, F., \& Burwitz, V. 2010i, ATel, 3013, 1

Pietsch, W., Lloyd, J., Henze, M., et al. 2010j, ATel, 2964, 1

Pietsch, W., Lloyd, J., Henze, M., et al. 2010k, ATel, 2896, 1

Pietsch, W., Podigachoski, P., Haberl, F., et al. 2009a, ATel, 2308, 1

Pietsch, W., Podigachoski, P., Haberl, F., et al. 2009b, CBET, 2061, 2

Podigachoski, P., Henze, M., Pietsch, W., et al. 2009a, ATel, 2234, 1

Podigachoski, P., Henze, M., \& Pietsch, W. 2009b, CBET, 1971, 1

Podigachoski, P., Pietsch, W., Henze, M., et al. 2009c, ATel, 2304, 1

Podigachoski, P., Pietsch, W., \& Henze, M. 2009d, CBET, 2058, 2

Prieto, J., Khan, R., Garnavich, P., Pagnini, J., \& Yusa, T. 2010a, CBET, 2594, 1

Prieto, J., Khan, R., \& Hornochova, P. 2010b, CBET, 2610, 2

Rau, A., Kulkarni, S. R., Law, N. M., et al. 2009, PASP, 121, 1334

Rector, T. A., Jacoby, G. H., Corbett, D. L., \& Denham, M. 1999, BAAS, 31, 1420

Rodríguez-Gil, P., Ferrando, R., Rodríguez, D., et al. 2009, ATel, 2166, 1

Romadan, A., Whallon, N., Zheng, W., et al. 2010, ATel, 2840, 1

Rosino, L. 1964, Ann. Astrophys., 27, 498

Rosino, L. 1973, A\&AS, 9, 347

Rosino, L., Capaccioli, M., D’Onofrio, M., \& della Valle, M. 1989, AJ, 97, 83

Schaefer, B. E. 2010, ApJS, 187, 275

Schlegel, D. J., Finkbeiner, D. P., \& Davis, M. 1998, ApJ, 500, 525

Shafter, A. W., Bode, M. F., Darnley, M. J., Ciardullo, R., \& Misselt, K. A. 2010a, ATel, 3006, 1

Shafter, A. W., Bode, M. F., Darnley, M. J., et al. 2011a, ApJ, 727, 50

Shafter, A. W., Ciardullo, R., Bode, M. F., Darnley, M. J., \& Misselt, K. A. 2010b, ATel, 2987, 1

Shafter, A. W., Ciardullo, R., Bode, M. F., Darnley, M. J., \& Misselt, K. A. 2010c, ATel, 2843, 1
Shafter, A. W., Ciardullo, R., Bode, M. F., Darnley, M. J., \& Misselt, K. A. 2010d, ATel, 2834, 1

Shafter, A. W., Ciardullo, R., Darnley, M. J., Bode, M. F., \& Misselt, K. A. 2010e, ATel, 3074, 1

Shafter, A. W., Ciardullo, R., Darnley, M. J., Bode, M. F., \& Misselt, K. A. 2010f, ATel, 2898, 1

Shafter, A. W., Ciardullo, R., Darnley, M. J., Bode, M. F., \& Misselt, K. A. 2010g, ATel, 2949, 1

Shafter, A. W., Ciardullo, R., Darnley, M. J., Bode, M. F., \& Misselt, K. A. 2010h, ATel, 2909, 1

Shafter, A. W., Darnley, M. J., Hornoch, K., et al. 2011b, ApJ, 734, 12

Shafter, A. W., Hornoch, K., Darnley, M. J., et al. 2010i, ATel, 3039, 1

Shafter, A. W., \& Irby, B. K. 2001, ApJ, 563, 749

Shafter, A. W., Rau, A., Quimby, R. M., et al. 2009, ApJ, 690, 1148

Shafter, A. W., \& Quimby, R. M. 2007, ApJ, 671, L121

Sharov, A. S., \& Alksnis, A. 1991, Ap\&SS, 180, 273

Shaviv, N. J. 2001, MNRAS, 326, 126

Siegel, M. H., Hoversten, E. A., Roming, P. W. A., et al. 2010, ApJ, 725, 1215

Starrfield, S., Sparks, W. M., \& Shaviv, G. 1988, ApJ, 325, L35

Strope, R. J., Schaefer, B. E., \& Henden, A. A. 2010, AJ, 140, 34

Sun, G., Wenzhou, Zhejiang, \& Gao, X. 2010, CBET, 2516, 3

Tamm, A., Tempel, E., \& Tenjes, P. 2007, arXiv:0707.4375

Tomaney, A. B., Crotts, A., \& Shafter, A. 1992, BAAS, 24, 1237

Tomaney, A. B., \& Shafter, A. W. 1992, ApJS, 81, 683

Trudolyubov, S., \& Priedhorsky, W. 2004, ApJ, 616, 821

Vilardell, F., Ribas, I., Jordi, C., Fitzpatrick, E. L., \& Guinan, E. F. 2010, A\&A, 509, A70

Williams, R., Mason, E., Della Valle, M., \& Ederoclite, A. 2008, ApJ, 685, 451

Wirth, A., Smarr, L. L., \& Bruno, T. L. 1985, ApJ, 290, 140

Yamaoka, H., Ayani, K., Honda, S., \& Kinugasa, K. 2009, CBET, 1980, 1

Yusa, T. 2010a, CBET, 2448, 1

Yusa, T. 2010b, CBET, 2472, 1

Yusa, T. 2010c, CBET, 2483, 1

Yusa, T. 2010d, CBET, 2582, 6

Yusa, T. 2010e, CBET, 2597, 1

Zheng, W., Romadan, A., Whallon, N., et al. 2010, CBET, 2574, 1 